\title{
A systematic review and meta-analysis of postpartum contraceptive use among women in low- and middle-income countries
}

\author{
Rubee Dev ${ }^{1 *}$, Pamela Kohler ${ }^{2}$, Molly Feder ${ }^{3}$, Jennifer A. Unger ${ }^{4}$, Nancy F. Woods ${ }^{5}$ and Alison L. Drake ${ }^{6}$
}

\begin{abstract}
Background: Short birth intervals increase risk for adverse maternal and infant outcomes including preterm birth, low birth weight (LBW), and infant mortality. Although postpartum family planning (PPFP) is an increasingly high priority for many countries, uptake and need for PPFP varies in low- and middle-income countries (LMIC). We performed a systematic review and meta-analysis to characterize postpartum contraceptive use, and predictors and barriers to use, among postpartum women in LMIC.

Methods: PubMed, EMBASE, CINAHL, PsycINFO, Scopus, Web of Science, and Global Health databases were searched for articles and abstracts published between January 1997 and May 2018. Studies with data on contraceptive uptake through 12 months postpartum in low- and middle-income countries were included. We used random-effects models to compute pooled estimates and confidence intervals of modern contraceptive prevalence rates $(\mathrm{mCPR})$, fertility intentions (birth spacing and birth limiting), and unmet need for contraception in the postpartum period.

Results: Among 669 studies identified, 90 were selected for full-text review, and 35 met inclusion criteria. The majority of studies were from East Africa, West Africa, and South Asia/South East Asia. The overall pooled mCPR during the postpartum period across all regions was $41.2 \%$ (95\% Cl: 15.7-69.1\%), with lower pooled mCPR in West Africa (36.3\%; 95\% Cl: 27.0-45.5\%). The pooled prevalence of unmet need was $48.5 \%$ (95\% Cl: 19.1-78.0\%) across all regions, and highest in South Asia/South East Asia (59.4, 95\% Cl: 53.4-65.4\%). Perceptions of low pregnancy risk due to breastfeeding and postpartum amenorrhea were commonly associated with lack of contraceptive use and use of male condoms, withdrawal, and abstinence. Women who were not using contraception were also less likely to utilize maternal and child health $(\mathrm{MCH})$ services and reside in urban settings, and be more likely to have a fear of method side effects and receive inadequate FP counseling. In contrast, women who received FP counseling in antenatal and/or postnatal care were more likely to use PPFP.
\end{abstract}

Conclusions: PPFP use is low and unmet need for contraception following pregnancy in LMIC is high. Tailored counseling approaches may help overcome misconceptions and meet heterogeneous needs for PPFP.

Keywords: Barriers, Contraceptives, Predictors, Postpartum, Low income, Middle income

\footnotetext{
* Correspondence: meetrubss@hotmail.com

'Sun Yat-sen Global Health Institute, Sun Yat-sen University, Guangzhou,

Guangdong, China

Full list of author information is available at the end of the article
}

(c) The Author(s). 2019 Open Access This article is distributed under the terms of the Creative Commons Attribution 4.0 International License (http://creativecommons.org/licenses/by/4.0/), which permits unrestricted use, distribution, and reproduction in any medium, provided you give appropriate credit to the original author(s) and the source, provide a link to the Creative Commons license, and indicate if changes were made. The Creative Commons Public Domain Dedication waiver (http://creativecommons.org/publicdomain/zero/1.0/) applies to the data made available in this article, unless otherwise stated. 


\section{Plain English summary}

This review was conducted to describe contraceptive use by women immediately after delivery through 1 year postpartum. Starting contraception after delivery is important to prevent unintended pregnancies and short birth intervals, which are related to adverse health outcomes for the mother and child. Despite desires to delay future pregnancy, many women in the studies reviewed did not use any contraceptive method (58.8\%) or used methods that provided short-term coverage with higher potential of failure (51-96\%). Contraceptive uptake was low, and need was high, in West Africa compared to South Asia/South East Asia and East Africa. The most commonly reported reasons for non-use of contraception were low perceived risk of getting pregnant and fear of side effects, while resumption of menses following delivery were commonly reported predictors of use. Receipt of family planning services in both antenatal and postnatal clinics, and appropriate contraceptive counseling, were also more frequently reported among contraceptive users. In contrast, lack of awareness on available methods and rural residence were more common among women who were not using family planning. Accurate counseling on returning fertility after childbirth, lactational amenorrhea and information on possible contraceptive side-effects may facilitate acceptance and use of contraception during the postpartum period. A tailored counseling approach that addresses women's needs and preferences could further reduce method dissatisfaction, discontinuation, and switching.

\section{Background}

Short birth intervals increase risks of adverse maternal and infant outcomes, such as low-birth weight and infant mortality $[1,2]$. Birth intervals shorter than 18 months have the highest mortality risk for infants and children under-five, with decreasing risk as birth intervals increase up to 36 months [3]. As a result, the World Health Organization (WHO) recommends birth intervals of 2-3 years [2]. Postpartum family planning (PPFP) or the postpartum contraception, defined as the initiation of contraceptive methods within the first 12 months following delivery $[4,5]$, can help women space their births, providing important maternal and child health $(\mathrm{MCH})$ benefits [6]. Spacing births by at least 2 years can reduce maternal mortality by $30 \%$ and child mortality by $10 \%$ [7].

The majority (91\%) of postpartum women in low- and middle-income countries (LMIC) report a desire to prevent pregnancy for at least a year following a birth [8]; yet, use of family planning (FP) methods reported previously is low [9-11] and risk of unintended pregnancy is high in the postpartum period [12-14]. Even among women who use modern FP methods, use of highly effective, long-acting reversible contraception
(LARC), including intra-uterine devices (IUDs) and implants, is low $(<15 \%)[15,16]$.

Individual studies suggest contraceptive use among postpartum women varies widely across geographical regions in LMIC $[8,13,16,17]$. However, differences in study design, temporal changes in contraceptive use, and variations in the definitions of unmet need have made it difficult to compare estimates of contraceptive use and unmet need between settings. In addition, individual, societal, and/or health systems factors affect uptake of contraception during the postpartum period contributing further to the variation.

Identifying similarities and differences in contraceptive use patterns, unmet need for PPFP, and factors that affect PPFP across low-resource settings is critical to informing strategies to enable women to effectively space and limit pregnancies and improve overall $\mathrm{MCH}$. We conducted a systematic review and meta-analysis to summarize modern contraceptive prevalence rates (mCPRs), fertility intentions, and unmet need among postpartum women in LMIC, which are our primary outcomes. We also reviewed barriers and facilitators to using contraception during the postpartum period, which are our secondary outcomes.

\section{Methods}

\section{Search strategy}

We conducted a search for all peer-reviewed published articles on PPFP using PubMed, EMBASE, PsycINFO, CINAHL, Scopus, Web of Science, and Global Health databases from January 1997 to May 2018. A combination of Medical Subject Headings or key search terms included: (postpartum OR post-delivery OR parturition OR puerperium) AND (use OR behavior OR preference OR barrier) AND (contraception OR contraceptive OR family planning) AND (resource limited OR low income OR middle income). We also conducted an internet search using the Google search engine to identify published online articles related to postpartum contraceptive use that may be excluded from these databases. Titles of articles without abstracts were reviewed for consideration in the full-text review; duplicate titles of articles were excluded from the review. Articles and proceedings of recent international meetings and conferences on PPFP (2014 International seminar on promoting postpartum and postabortion family planning, 2016 International Conference on Family Planning) were included in the full-text review if the abstract or title mentioned postpartum contraception or postpartum family planning.

\section{Inclusion and exclusion criteria}

The systematic review and meta-analysis included studies of postpartum women in LMIC based on the World Bank classification [18]. There were no restrictions on 
study design; experimental studies, observational studies, reviews, and reports were all eligible for inclusion. Data from postpartum women who used contraceptive methods within 12 months postpartum were included in the review; studies that report follow-up data beyond 12 months postpartum were included if data could be disaggregated to only include data during the first 12 months postpartum. Studies were included in the review or meta-analysis if one or more of the following outcomes were reported: $\mathrm{mCPR}$; unmet need for FP; and/or fertility intentions (birth spacing/limiting). Additionally, studies that included data on barriers or facilitators of contraceptive use were included in the review. We also included qualitative studies in the review to explore women's perspectives on contraceptive use. Articles were excluded if they were not in English or did not specify the duration of postpartum follow-up. Reports from Demographic and Health Surveys (DHS) were excluded since contraceptive use for postpartum women included women who delivered in the last 5 years without disaggregating the postpartum duration. However, secondary analyses of DHS data on postpartum women that include follow-up restrictions through 12 months postpartum were included. Unpublished articles and articles for which full-text could not be obtained were excluded. Authors of the original articles were not contacted to obtain any additional research data.

\section{Abstract review and quality assessment}

Articles and reports identified for review were imported into Covidence, a web-based software platform that streamlines citation review, resolution of discrepancies between independent reviewers, and agreement on final consensus data. All imported studies were initially reviewed for inclusion based on information contained in titles, keywords, and abstracts by two independent reviewers (RD and MF). The same two reviewers independently assessed the risk of bias of the studies using a modified version of the Newcastle-Ottawa Scale. Assessment was done based on sample representativeness, sample size, non-respondents, ascertainment of study outcomes, and quality of descriptive statistics reporting. Studies were judged to be at low risk of bias ( $\geq 3$ points) or high risk of bias (<3 points) [19]. Any unresolved disagreements between the two reviewers were discussed and consensus was reached by involving a third reviewer (ALD). All three reviewers (RD, MF, and ALD) were trained in epidemiology and conduct of reviews.

\section{Contraceptive definitions}

Postpartum contraceptive use was defined as using one or more of the following method(s): male or female condoms, spermicides, oral contraceptive pills [OCPs], injectables, implants, IUDs, sub-dermal implants, male and female sterilizations, emergency contraceptive pills [ECPs], lactational amenorrhea method [LAM], standard days method [SDM], rhythm/calendar method, withdrawal, or abstinence [20-22]. LARC was defined as the use of implants or IUDs [22]. Modern contraceptive methods was defined as using one or more of the following method(s): male and female condoms, OCPs, injectables, implants, IUDs, sub-dermal implants, male and female sterilizations, or ECPs; traditional methods was defined as using one or more of the following method(s): LAM, SDM, rhythm/calendar method, withdrawal, or abstinence [21].

The modern contraceptive prevalence rate ( $\mathrm{mCPR}$ ) was defined as the percentage of women who were currently using, or whose sexual partner was currently using, at least one method of modern contraception within the first year postpartum. mCPRs were classified according to the Track20 three stages of growth in 69 FP2020 focus countries; these are classified as low $(<20 \%)$, moderate $(20-$ $40 \%)$, and high (>40\%) [23, 24]. Fertility intentions were defined as women's desire for birth spacing or limiting. Postpartum women who delivered within last year and who wanted to postpone their next pregnancy for $\geq 2$ years were classified as desiring contraception for birth spacing, while women who did not want another child were classified as desiring contraception for birth limiting [25]. Unmet need for contraception among postpartum women in the studies included in the review was defined using prospective, retrospective, and current status definitions [26]. The prospective definition included women who did not want a child in the next 2 years but were not using modern contraceptives, including women who were amenorrhic or abstaining from sex; the retrospective definition included women who reported their prior pregnancy was unintended or unwanted [26, 27]; and the current status definition included women who had resumed sex and menses and were not using FP, but wanted to delay the next pregnancy for at least 2 years [26]. Only studies that used the prospective definition to assess unmet need were included in the meta-analysis due to the limited number of studies using the current status $(n=2)$ and retrospective $(n=1)$ definitions. Weighted means based on study sample size were calculated to summarize individual characteristics across studies.

\section{Pooled prevalence}

To account for study heterogeneity of $\mathrm{I}^{2}=99.9 \%$, which, according to Higgins et al. (2003) indicates the presence of high heterogeneity [28], we conducted a randomeffects meta-analysis [29]. Study locations were categorized into 4 regions: East Africa, West Africa, South Asia/South East Asia and Middle East/North Africa. We calculated pooled mCPR, unmet need for FP, and desire for birth spacing/limiting overall and stratified by region. We explored potential sources of heterogeneity in 
postpartum duration and temporal differences in study conduct using random-effects meta-regression [30, 31]. Timing of initiation of PPFP was dichotomized as $<6$ or $\geq 6$ months to align with recommendations for exclusively breastfeed through 6 months and ability to use the lactational amenorrhea method up to 6 months [32, 33]. Calendar year of study initiation was dichotomized as before or after 2012, which marks the 2012 London Summit calling for global commitments to expand access to contraception. If $95 \%$ confidence intervals (CIs) were not reported, standard errors were calculated to construct 95\% CIs [34]. Multi-country studies were disaggregated by country when possible. Meta-analyses and meta-regression were conducted using Stata version 14 (Stata corporation, College Station, TX, USA).

\section{Results}

\section{Studies selected for review}

Among 669 studies identified, 90 were selected for fulltext review, and 35 (34 articles and 1 seminar report) met inclusion criteria (Fig. 1). Characteristics of studies included in the review and meta-analysis are summarized in Table 1. Overall, 15 LMIC were included, representing a total of 74,001 postpartum women; the majority $(n=23)$ of studies were conducted in subSaharan Africa (8 in West Africa and 15 in East Africa), 7 in South Asia/South East Asia, 1 in Middle East/North Africa, and 4 in multiple regions. More than half $(n=25)$ were cross-sectional, with outcome ascertainments between 0 and 12 months postpartum. Most $(n=20)$ crosssectional studies in the review had outcomes ascertained at 12 months postpartum [9, 11, 16, 17, 35-40]. Among 6 prospective studies and one trial with follow-up, the weighted average duration of follow-up was 3 months postpartum. Among 16 studies that reported maternal age, the mean age of postpartum women was 28 years. Modified Newcastle-Ottawa risk of bias scores for all the individual studies included in the review and metaanalysis is presented in Table 1.

\section{Postpartum contraceptive use and behaviors Modern contraceptive prevalence rate (mCPR) between 0 and 12 months postpartum}

Postpartum mCPR was reported in 24 studies (Table 2), with an overall crude pooled estimate of $41.2 \%$ (95\% CI: $30.1-52.2, p<0.001)$ and similar in the adjusted analysis. mCPR was not associated with the timing of postpartum initiation $(p=0.95)$ or year the study was conducted $(p=0.41)$. Regionally, $\mathrm{mCPR}$ was highest in South Asia/South East Asia (42.4, 95\% CI: 15.7-69.1), followed by East Africa (39.5, 95\% CI: 28.2-50) (Fig. 2). Within these regions there was substantial variation in the mCPR. In South Asia/South East Asia mCPR ranged from $4.0 \%$ in Pakistan to $65.6 \%$ in India, while in East Africa it varied from 10.3\% in Ethiopia to 73.7\% in Uganda. Overall pooled mCPR was lowest in West Africa (36.3, 95\% CI: 27.0-45.5), and ranged from $25.5 \%$ in Ghana to $48.3 \%$ in Niger.

\section{Most commonly used contraceptive methods}

Contraceptive method-mix also varied widely across regions and countries. Among contraceptive users, the modern method most commonly initiated after birth was injectables, followed by OCPs and condoms. Injectables comprised the majority of the method-mix and ranged from $2.7 \%$ in Nigeria [41] to $68.5 \%$ in Ethiopia [10]. In Nigeria, women were less familiar with sterilization methods than other methods [41]. Twelve studies across all regions reported use of LARC was significantly lower than short-acting modern methods during the postpartum period $[8,10,16]$. However, LARC comprised a relatively larger proportion of the method-mix in Indonesia, Kenya, and Ethiopia. Use of LAM and the calendar method were commonly reported by women in West Africa, with 72.1 and $51.8 \%$ of women using these methods, respectively. Only two studies [36, 37] from Malawi in 2013 and Ghana in 2010 reported implants as the preferred method of choice after birth (Table 2).

\section{Fertility intentions of postpartum women}

Nine studies reported fertility intentions of postpartum women; eight reported birth limiting and six reported birth spacing. Across all regions, the pooled prevalence of desire for birth spacing was 54.8\% (95\% CI: $30.5-$ $79.2 \%$ ) (Fig. 3), which was higher than the pooled prevalence of desire for birth limiting (36.5, 95\% CI: 13.1-59.9\%) (Fig. 4) [9-11, 16, 42, 43]. Desire for birth spacing and birth limiting, independently, were significantly higher $(p<0.001)$ in South Asia/South East Asia (67.5\% for birth spacing and $58.0 \%$ for birth limiting) compared to East Africa (51.2\% for birth spacing and $31.7 \%$ for birth limiting). Regional differences for birth spacing versus birth limiting may be due to differences in the population age structure, with younger populations being more likely to favor birth spacing, as well as differences in desired family size.

\section{Unmet need for modern contraception}

Five studies representing 8 countries reported unmet need for contraception, which ranged from 16.3\% in Egypt to 96\% in Pakistan [8, 39, 41, 44, 45], highlighting high variability in risk of unintended pregnancy among women in LMICs. Overall, the pooled prevalence of unmet need was 48.5\% (95\% CI: 19.1-78.0\%) across all regions, and was highest in West Africa (59.4, 95\% CI: 53.4-65.4\%), followed by South Asia/South East Asia (58.4, 95\% CI: 8.1-108.7\%), and East Africa (45.6, 95\% CI: 28.4-62.8\%) (Fig. 5). Within South Asia, unmet need ranged from $31.6 \%$ in India to 


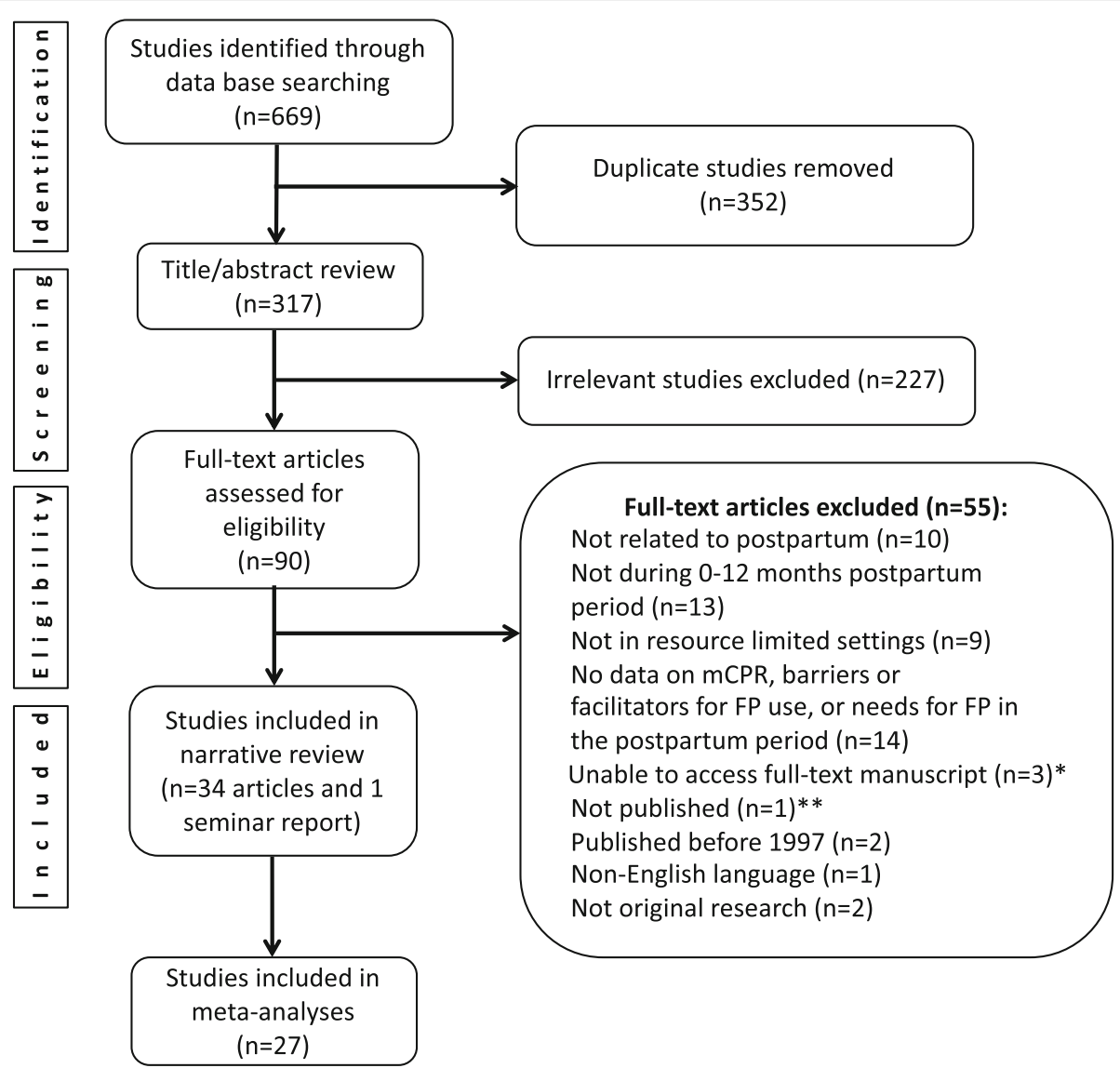

Fig. 1 PRISMA flow diagram of literature search results (Search Dates: January 1997-May 2018). mCPR; modern contraceptive prevalence rate, FP; family planning. * not indexed in electronic database at the time of review ${ }^{* *}$ unpublished dissertation

96.6\% in Pakistan, while in East Africa unmet need ranged from $25.5 \%$ in Zambia to $66.0 \%$ in Uganda. There was no relationship between timing of initiation of PPFP $(p=0.76)$, or year the study was conducted $(p=0.44)$ and unmet need for modern contraception.

Many reasons women who desire birth spacing or limiting but do not use a contraceptive method may be similar for postpartum women and non-postpartum women, but may also be specific to women who are breastfeeding. These reasons include fears, misconceptions, and cultural acceptability $[46,47]$.

\section{Facilitators for PPFP Facilitators and barriers for PPFP Demographic characteristics}

The proportion of women who use contraception declines with age; contraceptive use was highest among women < 24 and lowest among women $>35$ [10, 35, 38, 42, 48, 49]. Postpartum contraceptive use was also higher among women who were more educated $[11,35,40,43,44,48$, $50-56]$, lived in urban residence [17, 40, 43, 51, 52], and had higher socio-economic status $[40,49,51,52]$. However, in Sri Lanka contraceptive use was higher among women with low socio-economic status, which may be attributed to postnatal home visits by midwives who referred women for FP in this study [56]. In several studies, marital status and male partner support were associated with contraceptive use during the postpartum period [6, 11, 14, 16, 37, 44, 48, 50, 51, 57, 58]. Married women were consistently more likely to use contraception than single women, as were women who reported they had support from their partner to use FP $[6,11,57-60]$. In contrast, contraceptive use was lower among women without current partners, who may have less need for contraception due to lack of, or infrequent, sexual activity $[10,11]$. A summary of facilitators for contraceptive use in individual studies are shown in Fig. 6.

\section{Reproductive characteristics}

Resumption of menses, marking the return to fertility among postpartum women, has been shown to trigger contraceptive initiation among women in Tanzania, Kenya, and Ethiopia [10, 11, 17, 45, 50, 53, 60]. Women perceive pregnancy risks to be low when they are amenorrheic following delivery and during breastfeeding, despite the possibility of the return to fertility prior to resumption of menses. Two studies cite 
Table 1 Characteristics of studies on postpartum contraceptive use, by regions $(n=35)$

\begin{tabular}{|c|c|c|c|c|c|c|c|c|c|}
\hline \multirow{2}{*}{$\begin{array}{l}\text { First author } \\
\text { [Reference] }\end{array}$} & \multirow{2}{*}{$\begin{array}{l}\text { Publication } \\
\text { year }\end{array}$} & \multirow{2}{*}{$\begin{array}{l}\text { Survey } \\
\text { year(s) }\end{array}$} & \multirow{2}{*}{ Country } & \multirow[t]{2}{*}{ Study Design } & \multicolumn{3}{|c|}{ Study population } & \multirow{2}{*}{$\begin{array}{l}\text { Outcomes included } \\
\text { in the meta-analysis }\end{array}$} & \multirow{2}{*}{$\begin{array}{l}\text { New } \\
\text { Castle } \\
\text { Ottawa } \\
\text { Score }\end{array}$} \\
\hline & & & & & $\begin{array}{l}\text { Maternal } \\
\text { Age } \\
\text { (years) }^{\text {a }}\end{array}$ & $\begin{array}{l}\text { Time point of } \\
\text { assessment }\end{array}$ & $\begin{array}{l}\text { Sample } \\
\text { size }\end{array}$ & & \\
\hline \multicolumn{10}{|l|}{ West Africa } \\
\hline Adanikin [63] & 2013 & $\begin{array}{l}2011- \\
2012\end{array}$ & Nigeria & $\mathrm{RCT}$ & $\begin{array}{l}\text { Mean }= \\
29.2\end{array}$ & 6 months & 216 & $\mathrm{mCPR}$ & 4 \\
\hline Adeyemi [41] & 2005 & $\begin{array}{l}2003- \\
2004\end{array}$ & Nigeria & $\begin{array}{l}\text { Prospective } \\
\text { cohort }\end{array}$ & $\begin{array}{l}\text { Mean }= \\
28.5\end{array}$ & 9-10 months & 256 & mCPR, unmet need & 4 \\
\hline Eliason [6] & 2013 & 2012 & Ghana & $\begin{array}{l}\text { Cross- } \\
\text { sectional }\end{array}$ & $\begin{array}{l}\text { Mean = } \\
25.6\end{array}$ & At the clinic & 1914 & - & 4 \\
\hline Sipsma [51] & 2013 & 2006 & Niger & $\begin{array}{l}\text { Cross- } \\
\text { sectional }\end{array}$ & Mean $=29$ & 6 months & 673 & $\mathrm{mCPR}$ & 4 \\
\hline Robinson [37] & 2016 & 2010 & Ghana & $\begin{array}{l}\text { Qualitative } \\
\text { (FGD) }\end{array}$ & $\begin{array}{l}\text { Range }= \\
15-49\end{array}$ & 0-12 months & 13 & - & 2 \\
\hline $\begin{array}{l}\text { Durosinlorun } \\
\text { [62] }\end{array}$ & 2016 & $\begin{array}{l}2000- \\
2014\end{array}$ & Nigeria & $\begin{array}{l}\text { Retrospective } \\
\text { cohort }\end{array}$ & $\begin{array}{l}\text { Range }=< \\
20 \text { to } \geq 50\end{array}$ & 6 months & 5992 & - & 4 \\
\hline Iliyasu [53] & 2018 & 2015 & Nigeria & $\begin{array}{l}\text { Cross- } \\
\text { sectional }\end{array}$ & Mean $=27$ & 12 months & 317 & $\mathrm{mCPR}$ & 4 \\
\hline Morhe [54] & 2017 & 2011 & Ghana & $\begin{array}{l}\text { Cross- } \\
\text { sectional }\end{array}$ & $\begin{array}{l}\text { Mean = } \\
31.1\end{array}$ & 6-12 months & 200 & $\mathrm{mCPR}$ & 5 \\
\hline \multicolumn{10}{|l|}{ East Africa } \\
\hline Balkus [58] & 2007 & $\begin{array}{l}1999- \\
2003\end{array}$ & Kenya & $\begin{array}{l}\text { Prospective } \\
\text { cohort }\end{array}$ & $\begin{array}{l}\text { Range }= \\
18-42\end{array}$ & 12 months & 410 & $\mathrm{mCPR}$ & 3 \\
\hline Hubacher [42] & 2013 & $\begin{array}{l}2011- \\
2012\end{array}$ & Kenya & $\begin{array}{l}\text { Prospective } \\
\text { cohort }\end{array}$ & $\begin{array}{l}\text { Range }= \\
18-39\end{array}$ & $6-12$ weeks & 671 & $\begin{array}{l}\text { Birth spacing \& } \\
\text { limiting }\end{array}$ & 3 \\
\hline Mumah [35] & 2015 & $\begin{array}{l}2007- \\
2010\end{array}$ & Kenya & $\begin{array}{l}\text { Prospective } \\
\text { cohort }\end{array}$ & $\begin{array}{l}\text { Range }= \\
15-49\end{array}$ & 0-12 months & 3579 & mCPR, birth limiting & 4 \\
\hline Ndugwa [17] & 2011 & $\begin{array}{l}2007- \\
2008\end{array}$ & Kenya & $\begin{array}{l}\text { Prospective } \\
\text { cohort }\end{array}$ & $\begin{array}{l}\text { Range }= \\
11-52\end{array}$ & 0-12 months & 2994 & mCPR, birth limiting & 3 \\
\hline Abera [10] & 2015 & 2013 & Ethiopia & $\begin{array}{l}\text { Cross- } \\
\text { sectional }\end{array}$ & $\begin{array}{l}\text { Mean }= \\
27.2\end{array}$ & $\begin{array}{l}6 \text { weeks-12 } \\
\text { months }\end{array}$ & 703 & $\begin{array}{l}\text { mCPR, birth spacing \& } \\
\text { limiting }\end{array}$ & 5 \\
\hline Abraha [11] & 2017 & 2015 & Ethiopia & $\begin{array}{l}\text { Cross- } \\
\text { sectional }\end{array}$ & $\begin{array}{l}\text { Mean }= \\
27.4\end{array}$ & 0-12 months & 590 & $\begin{array}{l}\text { mCPR, birth spacing \& } \\
\text { limiting }\end{array}$ & 5 \\
\hline O'Shea [36] & 2014 & 2013 & Malawi & $\begin{array}{l}\text { Cross- } \\
\text { sectional }\end{array}$ & $\begin{array}{l}\text { Range }= \\
18-35+\end{array}$ & $0-12$ months & 634 & - & 3 \\
\hline Keogh [50] & 2015 & 2008 & Tanzania & $\begin{array}{l}\text { Cross- } \\
\text { sectional }\end{array}$ & $\begin{array}{l}\text { Range = } \\
15-35+\end{array}$ & 6-12 months & 5284 & $\begin{array}{l}\text { mCPR, birth spacing \& } \\
\text { limiting }\end{array}$ & 3 \\
\hline $\begin{array}{l}\text { Mengesha } \\
{[43]}\end{array}$ & 2015 & 2012 & Ethiopia & $\begin{array}{l}\text { Cross- } \\
\text { sectional }\end{array}$ & $\begin{array}{l}\text { Mean }= \\
28.3\end{array}$ & 12 months & 899 & $\begin{array}{l}\text { mCPR, birth spacing \& } \\
\text { limiting }\end{array}$ & 5 \\
\hline Shabiby [57] & 2015 & 2012 & Kenya & $\begin{array}{l}\text { Cross- } \\
\text { sectional }\end{array}$ & Mean $=26$ & $\begin{array}{l}\text { At discharge } \\
\text { after birth }\end{array}$ & 185 & - & 3 \\
\hline Sileo [44] & 2015 & 2012 & Uganda & $\begin{array}{l}\text { Cross- } \\
\text { sectional }\end{array}$ & $\begin{array}{l}\text { Mean }= \\
25.8\end{array}$ & 3 months & 258 & mCPR, unmet need & 4 \\
\hline MCHIP [39] & 2012 & $\begin{array}{l}2008- \\
2009\end{array}$ & Kenya & $\begin{array}{l}\text { Cross- } \\
\text { sectional } \\
\text { (DHS data) }\end{array}$ & $\begin{array}{l}\text { Range }= \\
15-49\end{array}$ & 0-12 months & 2264 & $\begin{array}{l}\text { Unmet need, birth } \\
\text { spacing \& limiting }\end{array}$ & 3 \\
\hline Achwoka [55] & 2017 & 2013 & Kenya & $\begin{array}{l}\text { Cross- }^{-} \\
\text {sectional }\end{array}$ & $\begin{array}{l}\text { Mean = } \\
25.8\end{array}$ & 8-10 months & 955 & $\mathrm{mCPR}$ & 5 \\
\hline $\begin{array}{l}\text { Gebremariam } \\
\text { [59] }\end{array}$ & 2017 & 2015 & Ethiopia & $\begin{array}{l}\text { Cross- } \\
\text { sectional }\end{array}$ & $\begin{array}{l}\text { Mean = } \\
30.8\end{array}$ & 6-12 months & 605 & $\mathrm{mCPR}$ & 5 \\
\hline $\begin{array}{l}\text { Gebremedhin } \\
{[60]}\end{array}$ & 2018 & 2015 & Ethiopia & $\begin{array}{l}\text { Cross- } \\
\text { sectional }\end{array}$ & $\begin{array}{l}\text { Range }= \\
15-49\end{array}$ & 12 months & 803 & $\mathrm{mCPR}$ & 4 \\
\hline
\end{tabular}


Table 1 Characteristics of studies on postpartum contraceptive use, by regions $(n=35)$ (Continued)

\begin{tabular}{|c|c|c|c|c|c|c|c|c|c|}
\hline \multirow{2}{*}{$\begin{array}{l}\text { First author } \\
\text { [Reference] }\end{array}$} & \multirow{2}{*}{$\begin{array}{l}\text { Publication } \\
\text { year }\end{array}$} & \multirow{2}{*}{$\begin{array}{l}\text { Survey } \\
\text { year(s) }\end{array}$} & \multirow[t]{2}{*}{ Country } & \multirow[t]{2}{*}{ Study Design } & \multicolumn{3}{|c|}{ Study population } & \multirow{2}{*}{$\begin{array}{l}\text { Outcomes included } \\
\text { in the meta-analysis }\end{array}$} & \multirow{2}{*}{$\begin{array}{l}\text { New } \\
\text { Castle } \\
\text { Ottawa } \\
\text { Score }^{b}\end{array}$} \\
\hline & & & & & $\begin{array}{l}\text { Maternal } \\
\text { Age } \\
\text { (years) }^{a}\end{array}$ & $\begin{array}{l}\text { Time point of } \\
\text { assessment }\end{array}$ & $\begin{array}{l}\text { Sample } \\
\text { size }\end{array}$ & & \\
\hline Chhabra [13] & 2016 & 2014 & India & $\begin{array}{l}\text { Cross- } \\
\text { sectional }\end{array}$ & $\begin{array}{l}\text { Range }= \\
15-40\end{array}$ & 8 weeks & 117 & $\mathrm{mCPR}$ & 2 \\
\hline Kashyap [14] & 2016 & 2015 & India & $\begin{array}{l}\text { Cross- } \\
\text { sectional }\end{array}$ & $\begin{array}{l}\text { Range }= \\
18-35\end{array}$ & 10 weeks & 178 & $\mathrm{mCPR}$ & 2 \\
\hline Mody [48] & 2014 & 2008 & India & $\begin{array}{l}\text { Cross- } \\
\text { sectional }\end{array}$ & $\begin{array}{l}\text { Range }= \\
17-45\end{array}$ & 6 months & 1049 & $\mathrm{mCPR}$ & 3 \\
\hline Withers [38] & 2010 & $\begin{array}{l}2002- \\
2003\end{array}$ & Indonesia & $\begin{array}{l}\text { Cross- } \\
\text { sectional }\end{array}$ & $\begin{array}{l}\text { Mean }= \\
29.9\end{array}$ & 0-12 months & 1528 & $\mathrm{mCPR}$ & 5 \\
\hline $\begin{array}{l}\text { FP seminar } \\
{[40]}\end{array}$ & 2014 & NA & India & $\begin{array}{l}\text { Seminar } \\
\text { report }\end{array}$ & NS & $0-12$ months & $\begin{array}{l}56 \\
\text { countries }\end{array}$ & - & 2 \\
\hline Navodani [56] & 2017 & 2014 & Sri Lanka & $\begin{array}{l}\text { Cross- } \\
\text { sectional }\end{array}$ & $\begin{array}{l}\text { Mean }= \\
29.4\end{array}$ & 8-12 weeks & 1112 & $\mathrm{mCPR}$ & 5 \\
\hline Wilopo [49] & 2017 & 2015 & Indonesia & $\begin{array}{l}\text { Cross- } \\
\text { sectional }\end{array}$ & $\begin{array}{l}\text { Range }= \\
15-49\end{array}$ & 6 months & 1415 & mCPR, unmet need & 4 \\
\hline \multicolumn{10}{|c|}{ Middle East/North Africa } \\
\hline Elweshahi [45] & 2018 & 2016 & Egypt & $\begin{array}{l}\text { Cross- } \\
\text { sectional }\end{array}$ & Mean $=30$ & 12 months & 1500 & mCPR, unmet need & 5 \\
\hline \multicolumn{10}{|c|}{ Multi-regional (South Asia/Sub-Saharan Africa/Central America) } \\
\hline Moore [16] & 2015 & $\begin{array}{l}2005- \\
2012\end{array}$ & 21 LMIC & $\begin{array}{l}\text { Cross- } \\
\text { sectional } \\
\text { (DHS data) }\end{array}$ & $\begin{array}{l}\text { Range }= \\
15-49\end{array}$ & $0-12$ months & $\begin{array}{l}21 \\
\text { countries }\end{array}$ & - & 3 \\
\hline Ross [9] & 2001 & $\begin{array}{l}1991- \\
1996\end{array}$ & 27 countries & $\begin{array}{l}\text { Cross- } \\
\text { sectional } \\
\text { (DHS data) }\end{array}$ & $\begin{array}{l}\text { Range }= \\
15-49\end{array}$ & $0-12$ months & $\begin{array}{l}27 \\
\text { countries }\end{array}$ & - & 3 \\
\hline Pasha [8] & 2015 & $\begin{array}{l}2011- \\
2012\end{array}$ & $\begin{array}{l}\text { India, Pakistan, } \\
\text { Zambia, Kenya, } \\
\text { Guatemala }\end{array}$ & $\begin{array}{l}\text { Prospective } \\
\text { cohort }\end{array}$ & $\begin{array}{l}\text { Range }=< \\
20 \text { to } \geq 30\end{array}$ & 6 weeks & 36,687 & $\begin{array}{l}\text { mCPR, unmet need, } \\
\text { birth spacing \& } \\
\text { limiting }\end{array}$ & 3 \\
\hline Hounton [52] & 2015 & $\begin{array}{l}2004- \\
2013\end{array}$ & $\begin{array}{l}\text { Ethiopia, Malawi, and } \\
\text { Nigeria }\end{array}$ & $\begin{array}{l}\text { Cross- } \\
\text { sectional } \\
\text { (DHS data) }\end{array}$ & $\begin{array}{l}\text { Range }= \\
15-49\end{array}$ & 3 months & $\begin{array}{l}3 \\
\text { countries }\end{array}$ & - & 3 \\
\hline
\end{tabular}

Note: DHS (demographic and health survey), FGD (focus group discussion), MCHIP (maternal and child health integrated program), NA (not applicable), NS (not specified), PP (postpartum period),

$R C T$ (randomized controlled trial)

age at enrollment

${ }^{\mathrm{b}}$ Total modified Newcastle-Ottawa risk of bias scores for the studies included in this systematic review and meta-analysis. Scoring were done based on the (i) sample representativeness; (ii) sample size; (iii) non-respondents, (iv) ascertainment of $\mathrm{mCPR} /$ reproductive intention/unmet need; and (v) quality of descriptive statistics reporting. Total scores range from 0 to 5 . For the total score grouping, studies were judged to be of low risk of bias ( $\geq 3$ points) or high risk of bias $(<3$ points)

breastfeeding as the most frequent reason given by women for non-use of contraception [16, 38]. Women were found to avoid using hormonal contraception while breastfeeding due to a belief that hormonal methods could reduce milk production, and transfer hormones into milk that may harm the infant $[61,62]$. In several studies, contraceptive use was also significantly higher among women who reported wanting to limit or space their next births compared to women who reported having a desire for more children $[8,37$, $38,50]$. Some studies did show counseling in either ANC $[10,63]$ or PNC $[14,43]$ also improved contraceptive use, but results were not as robust as when counseling was offered in both settings $[11,40]$.

\section{Facility-based health services}

In our review, delivering at a health facility was a strong predictor of postpartum contraceptive use $[8,40,41,43,51$, 52]. Contraceptive counseling during $\mathrm{MCH}$ care was another important predictor of PPFP, but only when women were counseled during both antenatal and postpartum care $[11,40]$. However, multiple contraceptive counseling sessions in ANC/PNC were not found to increase PPFP use in one study conducted in Ghana [54]. Poor quality of counseling services may explain these discrepant results, since several studies reported that women received inadequate information during FP counseling, incomplete counseling, inadequate counseling on safety and efficacy of LARC, or misinformation on FP from providers [13, 37, 40, 51]. 
Table 2 Contraceptive use and need for postpartum family planning

\begin{tabular}{|c|c|c|c|c|}
\hline $\begin{array}{l}\text { Country, Year } \\
\text { published } \\
\text { [Reference] }\end{array}$ & N & mCPR $(95 \%$ Cl) & $\begin{array}{l}\text { Share of modern contraceptive } \\
\text { method-mix used postpartum (\%) }\end{array}$ & Fertility intention \& Unmet need (\%) \\
\hline \multicolumn{5}{|c|}{ Low mCPR (<20\%) } \\
\hline $\begin{array}{l}\text { Ethiopia, } \\
2015 \text { [43] }\end{array}$ & 899 & 10.3 & $\begin{array}{l}\text { Injectables (77.1), IUD (16.6) } \\
\text { OCP (3.1), Implant (2.1), Condom (1.1) }\end{array}$ & $\begin{array}{l}\text { Desire to space (7.1), Desire to limit (3.1), Unmet } \\
\text { need }(10.2)^{\mathrm{e}}\end{array}$ \\
\hline $\begin{array}{l}\text { Kenya, } \\
2013[42]\end{array}$ & 671 & - & $\begin{array}{l}\text { Injectables (36.4), Implant (30.1) } \\
\text { LNG-IUS (16.2), POP (14.7), IUD (2.6) }\end{array}$ & $\begin{array}{l}\text { Desire to limit }(25.5) \\
\text { Unmet need }(42.3)^{f}\end{array}$ \\
\hline \multicolumn{5}{|c|}{ Moderate mCPR (20-40\%) } \\
\hline $\begin{array}{l}\text { Niger, } \\
2013[51]\end{array}$ & 673 & 25.0 & $\begin{array}{l}\text { Among lactating women }{ }^{a} \\
\text { Modern methods }(25.0)^{b} \\
\text { Sterilizations (23.0) }\end{array}$ & - \\
\hline $\begin{array}{l}\text { Ghana, } \\
2017[54]\end{array}$ & & 25.5 & $\begin{array}{l}\text { Injectables (41.6), OCPs (15.1) } \\
\text { Condoms (15.1), Implants (9.4) } \\
\text { IUDs (9.4), Sterilization (9.4) }\end{array}$ & - \\
\hline $\begin{array}{l}21 \mathrm{LMIC}, \\
2015[16]\end{array}$ & - & 27.0 & Short-acting methods $(51.0-96.0)^{\mathrm{b}}$ & $\begin{array}{l}\text { Desire to space (37.0), Desire to limit (25.0), Unmet } \\
\text { need }(62.0)^{\mathrm{e}}\end{array}$ \\
\hline $\begin{array}{l}\text { Nigeria, } \\
2005[41]\end{array}$ & 256 & 29.7 & $\begin{array}{l}\text { Condoms (42.1), IUCD ( } 35.5 \text { ) } \\
\text { Pills (9.2), Injectables (9.2), Sterilization (4.0) }\end{array}$ & Unmet need $(59.4)^{\mathrm{e}}$ \\
\hline $\begin{array}{l}27 \text { countries, } \\
2001[9]\end{array}$ & & 30.0 & Pills (mainly in 0-6 months) ${ }^{b}$ & $\begin{array}{l}\text { Desire to space (39.1), Desire to limit (25.5), Unmet } \\
\text { need }(64.6 \% \text { across countries) })^{\text {e }}\end{array}$ \\
\hline $\begin{array}{l}\text { Tanzania, } \\
2015[50]\end{array}$ & 5284 & 34.0 & $\begin{array}{l}\text { Injectables (35.3), Condoms (29.4) } \\
\text { OCP (20.6), Dual method (14.7) }\end{array}$ & $\begin{array}{l}\text { Desire to space (11.0), Desire to limit (27.0), Unmet } \\
\text { need (38.0) }\end{array}$ \\
\hline $\begin{array}{l}\text { India, } \\
2014[48]\end{array}$ & 1049 & 33.6 & $\begin{array}{l}\text { Condoms (80.3), OCP (11.5) } \\
\text { IUD (5.1), Sterilization (2.5), ECP (0.6) }\end{array}$ & - \\
\hline $\begin{array}{l}\text { Nigeria, } \\
2013[63]\end{array}$ & $108^{c}$ & 35.4 & $\begin{array}{l}\text { Condoms (51.4), IUD (31.5), OCP (11.4), } \\
\text { Injectables (5.7) }\end{array}$ & - \\
\hline $\begin{array}{l}\text { India, } \\
2016[13]\end{array}$ & 117 & $36.0^{f}$ & IUCD (28.6), POP (14.2), Injectables (7.1) & Unmet need $(25.6)^{f}$ \\
\hline $\begin{array}{l}\text { Kenya, } \\
2011[17]\end{array}$ & 2264 & 36.0 & - & Unmet need $(59.0)^{\mathrm{e}}$ \\
\hline \multicolumn{5}{|c|}{ High mCPR (> 40\%) } \\
\hline $\begin{array}{l}\text { Indonesia, } \\
2010[38]\end{array}$ & 1528 & 40.5 & $\begin{array}{l}\text { Injectables (52.6), Implants (28.7) } \\
\text { IUD (9.5), OCPs (5.3), Sterilization (3.9) }\end{array}$ & Unmet need $(41.0)^{\mathrm{e}}$ \\
\hline $\begin{array}{l}\text { Nigeria, } \\
2018[53]\end{array}$ & & 41.6 & $\begin{array}{l}\text { Injectables (34.8), OCPs (21.2), IUDs (11.3), } \\
\text { Condoms (6.8), Sterilization (3.0) }\end{array}$ & - \\
\hline $\begin{array}{l}\text { Kenya, } \\
2011[17]\end{array}$ & 2994 & 43.2 & $\begin{array}{l}\text { Injectables (48.0), Pills (22.0) } \\
\text { Condoms (6.0) }\end{array}$ & Desire to limit (32.2) \\
\hline $\begin{array}{l}\text { India, } \\
2016[14]\end{array}$ & 178 & 44.0 & IUD, POP, Injectables ${ }^{\mathrm{b}}$ & - \\
\hline $\begin{array}{l}\text { Nigeria, } \\
2016 \text { [62] }\end{array}$ & 2924 & $47.6^{9}$ & Injectables (45.9), IUDs (36.8), OCP (12.7) & - \\
\hline $\begin{array}{l}\text { Ethiopia, } \\
2017[11]\end{array}$ & 590 & $48.0(43.9-52.2)$ & $\begin{array}{l}\text { Injectables (59.7), Implants (24.7) } \\
\text { Pills (12.0) }\end{array}$ & Desire to space (67.1), Desire to limit (14.7) \\
\hline $\begin{array}{l}\text { Ethiopia, } \\
2015[10]\end{array}$ & 703 & $48.4(44.5-52.1)$ & Injectables (68.5), OCPs (16.8) & Desire to space (51.1), Desire to limit (46.1) \\
\hline $\begin{array}{l}\text { Kenya, } \\
2015[35]\end{array}$ & 3579 & $\begin{array}{l}49.0-\text { in } 6 \text { months } \\
60.0 \text { - in } 12 \text { months }\end{array}$ & (Injectables, Pills) $)^{b}$, Condoms (6.0) & Desire to limit (32.1) \\
\hline $\begin{array}{l}\text { Malawi, } \\
2014[36]\end{array}$ & 634 & - & $\begin{array}{l}\text { Methods planned }^{\text {a }} \\
\text { Implant (67.0), Condom (42.0), } \\
\text { Injectables (38.0) }\end{array}$ & Desire to limit (97.0) \\
\hline $\begin{array}{l}\text { Indonesia, } \\
2017 \text { [49] }\end{array}$ & & 50 & $\begin{array}{l}\text { Injectables (71.2), OCPs (8.8) } \\
\text { IUDs (5.9), Implants (3.5) } \\
\text { Sterilization (5.3) }\end{array}$ & Unmet need (47) \\
\hline Kenya, 2017 & 955 & 59 & Injectables (64.4), Implants (16.9) & Unmet need (34) \\
\hline
\end{tabular}


Table 2 Contraceptive use and need for postpartum family planning (Continued)

\begin{tabular}{|c|c|c|c|c|}
\hline $\begin{array}{l}\text { Country, Year } \\
\text { published } \\
\text { [Reference] }\end{array}$ & $\mathrm{N}$ & mCPR $(95 \% \mathrm{Cl})$ & $\begin{array}{l}\text { Share of modern contraceptive } \\
\text { method-mix used postpartum }(\%)^{\Phi}\end{array}$ & Fertility intention \& Unmet need (\%) \\
\hline$[55]$ & & & $\begin{array}{l}\text { OCPs (10.2), IUDs (3.4), Condoms (3.4), } \\
\text { Sterilization (1.7) }\end{array}$ & \\
\hline $\begin{array}{l}\text { Sri Lanka, } \\
2017[56]\end{array}$ & 1112 & 64.5 & $\begin{array}{l}\text { Condoms (30.9), IUDs (27.2) } \\
\text { Injectables (23.3), OCPs (0.8) }\end{array}$ & - \\
\hline $\begin{array}{l}\text { Ethiopia, } \\
2017[59]\end{array}$ & 605 & $68.1(64.4-71.8)$ & $\begin{array}{l}\text { Injectables (58.8), Implants (31.8) } \\
\text { Pills (4.9), IUDs (3.4), Sterilization (0.1) }\end{array}$ & \\
\hline $\begin{array}{l}\text { Kenya, } \\
2007[58]\end{array}$ & $319^{d}$ & $72.0^{\mathrm{e}}$ & $\begin{array}{l}\text { Condoms (65.0) }{ }^{a} \text {, OCPs (31.0) Injectables (44.0), } \\
\text { Switched methods (25) }\end{array}$ & - \\
\hline $\begin{array}{l}5 \text { LMIC, } \\
2015[8]\end{array}$ & $\begin{array}{l}36, \\
687\end{array}$ & $\begin{array}{l}\text { Zambia (73.5), India } \\
\text { (65.5), Pakistan (4.0) }\end{array}$ & OCP or injectables $(>90)$, LARC $(3.0-10.0)^{b}$ & Unmet need $(25.0-96.0)^{\mathrm{e}}$ \\
\hline $\begin{array}{l}\text { Ethiopia, } \\
2018 \text { [60] }\end{array}$ & & $80.3(74.5-83.1)^{\#}$ & $\begin{array}{l}\text { Injectables (34.2), OCPs (22.2) } \\
\text { Implants (27.3), IUDs (7), Condoms (2.1) }\end{array}$ & \\
\hline $\begin{array}{l}\text { Egypt, } \\
2018[45]\end{array}$ & 1500 & 80.7 & - & Unmet need (16.3) \\
\hline \multicolumn{5}{|c|}{ 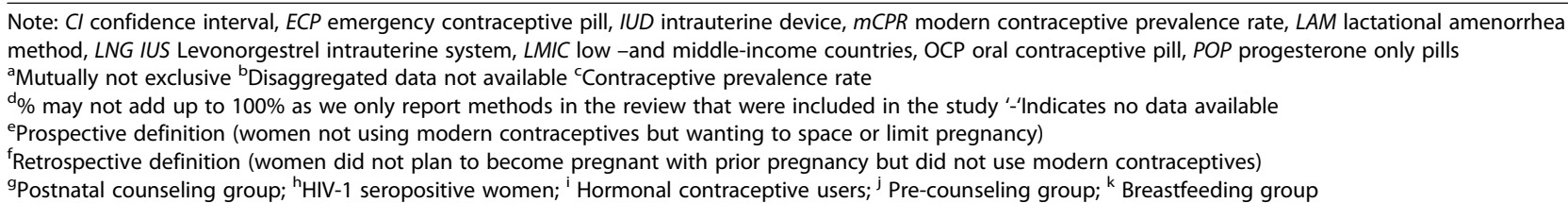 } \\
\hline
\end{tabular}

\section{Contraception acceptability and availability}

Fear of side-effects was identified in most studies as a reason for not using contraception during the postpartum period [11, 16, 37, 41, 42]. Women feared excessive bleeding, impact on milk supply, migraines, and weight gain. In addition, they feared pain, injury and discomfort associated with IUD and implant insertion, as well as inconvenience of IUD insertion [11, 16, 37, 40-42, 45, 57, 58]. Contraceptive methods that were perceived to be more convenient to use $[6,62]$, could be used confidentially [42], more familiar to women [36, 37, 41], and easier to access [9, 13, 40] were more acceptable to postpartum women. Inconsistent supply and stock-out of contraceptive products [13, 40], and poor accessibility of contraceptive information or lack of awareness of contraceptive methods, [36, 41] were also described as barriers to using PPFP.

\section{Perceptions of pregnancy risk and prior experience}

Concerns about pregnancy risk and potential benefits of contraception were major reasons cited for using PPFP. Similarly, low perceptions of pregnancy risk and fear of future infertility $[10,17,41]$ were reasons reported for non-use. Women who have previously used contraception have been shown to be more likely to use contraception in the postpartum period, and prior experience with contraception was an important predictor of postpartum contraceptive use in studies in our review [6, 42, $44,50,60]$. In Uganda, woman who had used contraception prior to the most recent pregnancy were $80 \%$ more likely to use contraceptive methods than women without contraceptive experience (adjusted Odds Ratio [aOR] =
1.8, 95\% CI: 1.36-2.37) [44]. Similarly, women who had previously had negative experiences with contraception were more reluctant to use contraception again. One study in Ethiopia found women who experienced problems their contraceptive method before their last pregnancy were $64 \%$ less likely use contraception in the postpartum period $(\mathrm{aOR}=0.34,95 \% \mathrm{CI}: 0.16-0.72)$ [11].

\section{Socio-cultural factors}

Religious and cultural factors also influence acceptance, and use, of contraception during the postpartum period. Two studies in the review described cultural norms encouraging childbearing and/or religious prohibition of contraception for postpartum women [36, 40]. Differences in the desired number of children varies regionally, which may partially explain the regional differences between parity and contraceptive use. In Kenya, women with higher parity ( $\geq$ 4) were less likely to utilize contraception than women with lower parity in the postpartum period $[35,52]$. In contrast, postpartum women in South East Asia were more likely to use contraception if they had more children [38, 48].

\section{Discussion}

In our meta-analysis, the overall pooled estimate of mCPR in the year following birth was low $(41.2$, 95\% CI: 30.1$52.2 \%)$; however, use varied regionally with higher $\mathrm{mCPR}$ in East and South Africa and lower $\mathrm{mCPR}$ in West Africa. Pooled desire for birth spacing (54.8\%) or birth limiting (36.5\%) were high among women, yet unmet need was also high. Our findings suggest there are substantial gaps in 


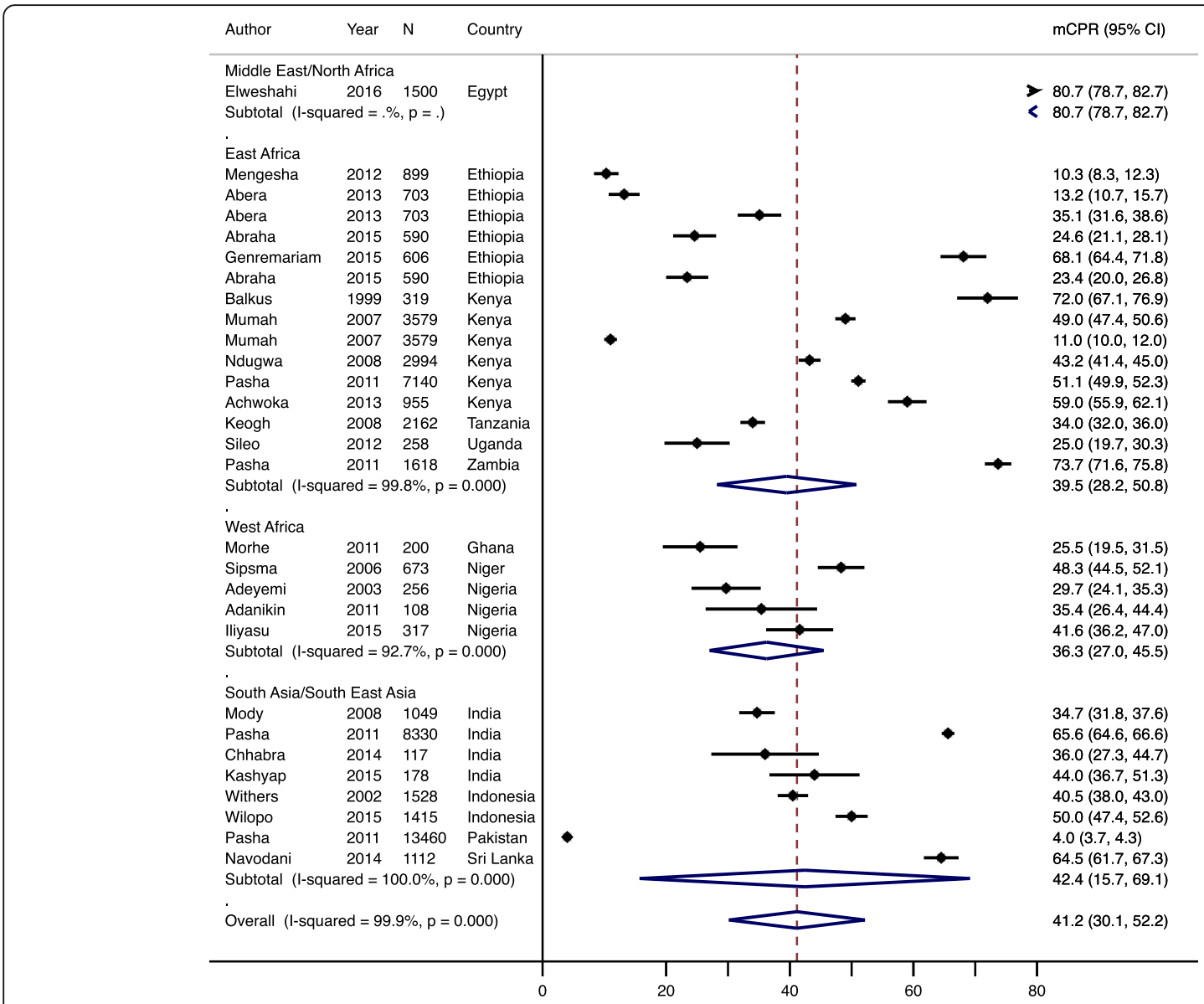

Fig. 2 Forest plot of modern contraceptive prevalence rate, by region. Year is start of survey year and $\mathrm{N}$ is sample size. mCPR; modern contraceptive prevalence rate, $\mathrm{Cl}$; confidence interval

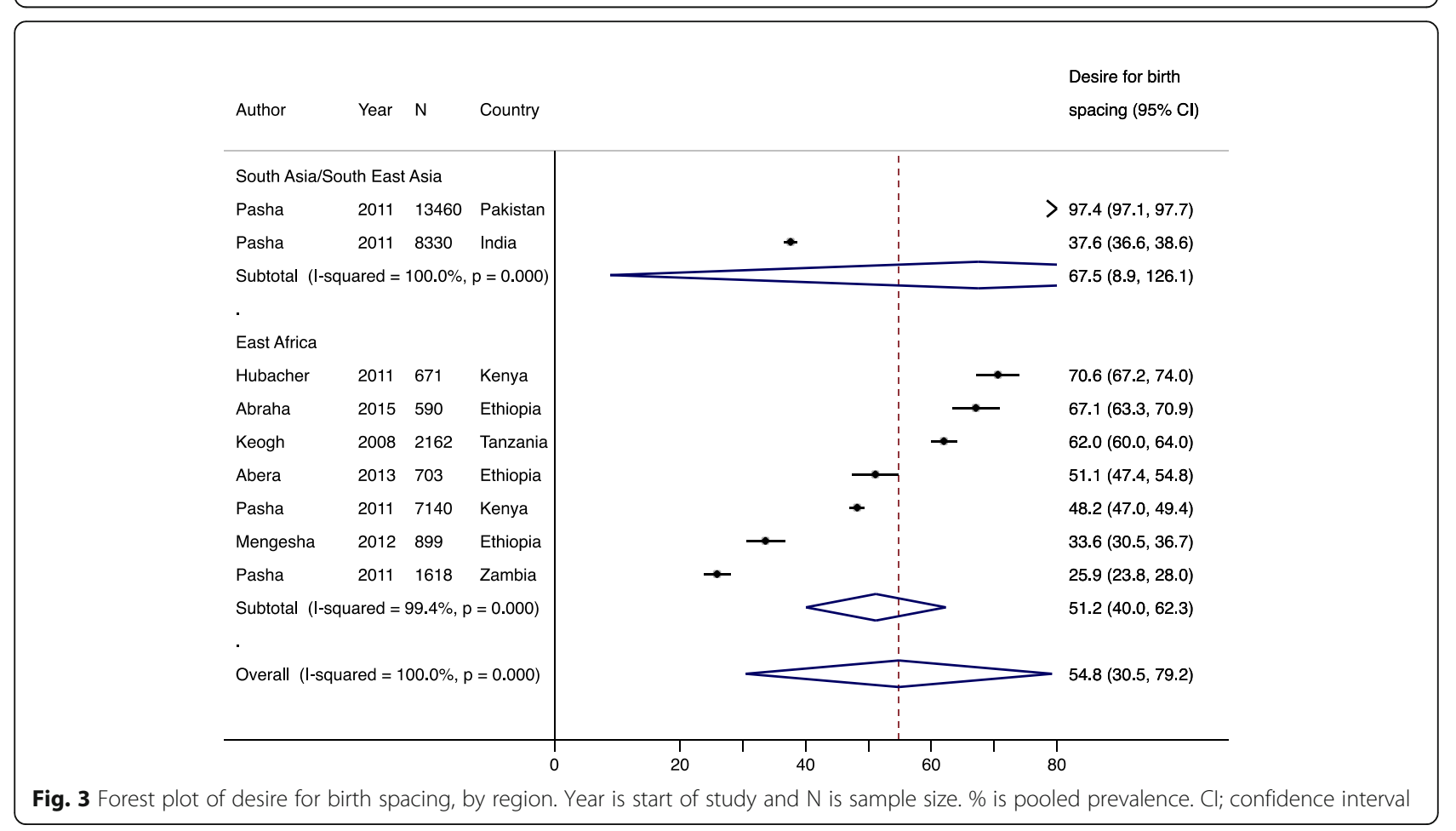




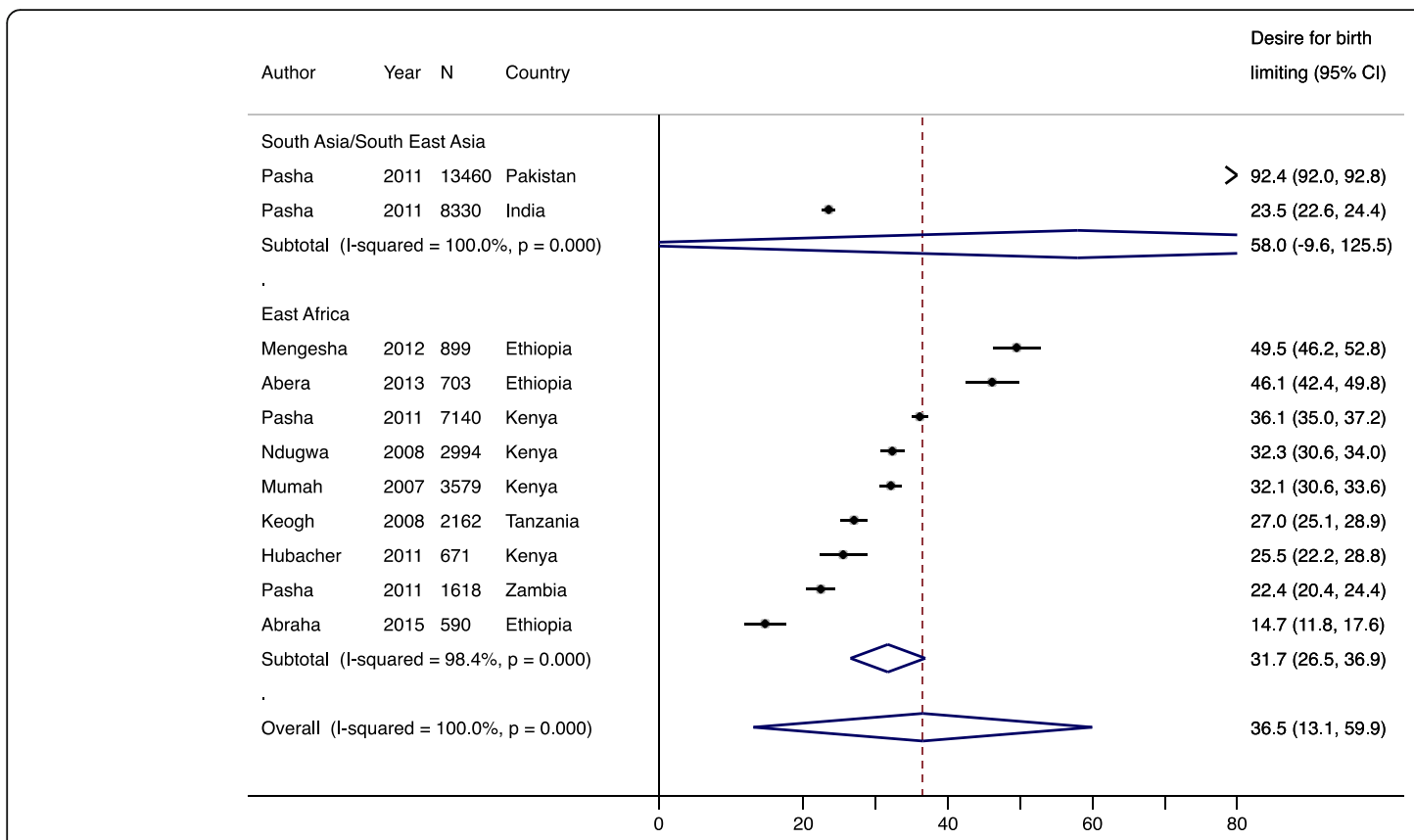

Fig. 4 Forest plot of desire for birth limiting, by region. Year is start of study and $\mathrm{N}$ is sample size. \% is pooled prevalence. Cl; confidence interval

helping women achieve their desired family size by delaying or preventing future pregnancies through contraception during the postpartum period.

The regional variations in contraceptive use and unmet need may be attributed to specific factors related to the postpartum period and breastfeeding, as well as patterns of contraceptive use among all women in these different regions. In sub-Saharan Africa, low PPFP use may be related to heterogeneous social and cultural beliefs. These include traditional practices of postpartum abstinence and reliance

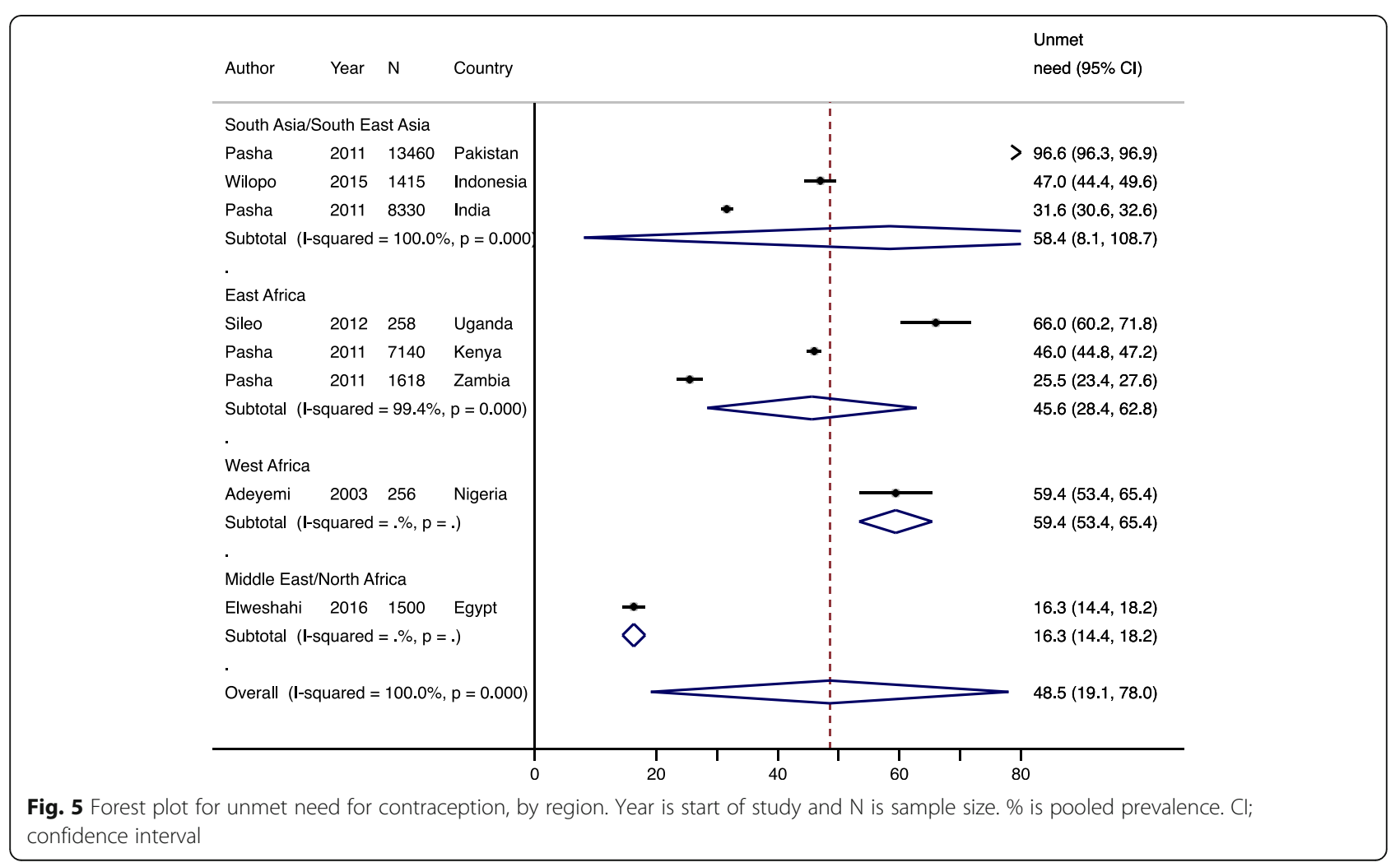




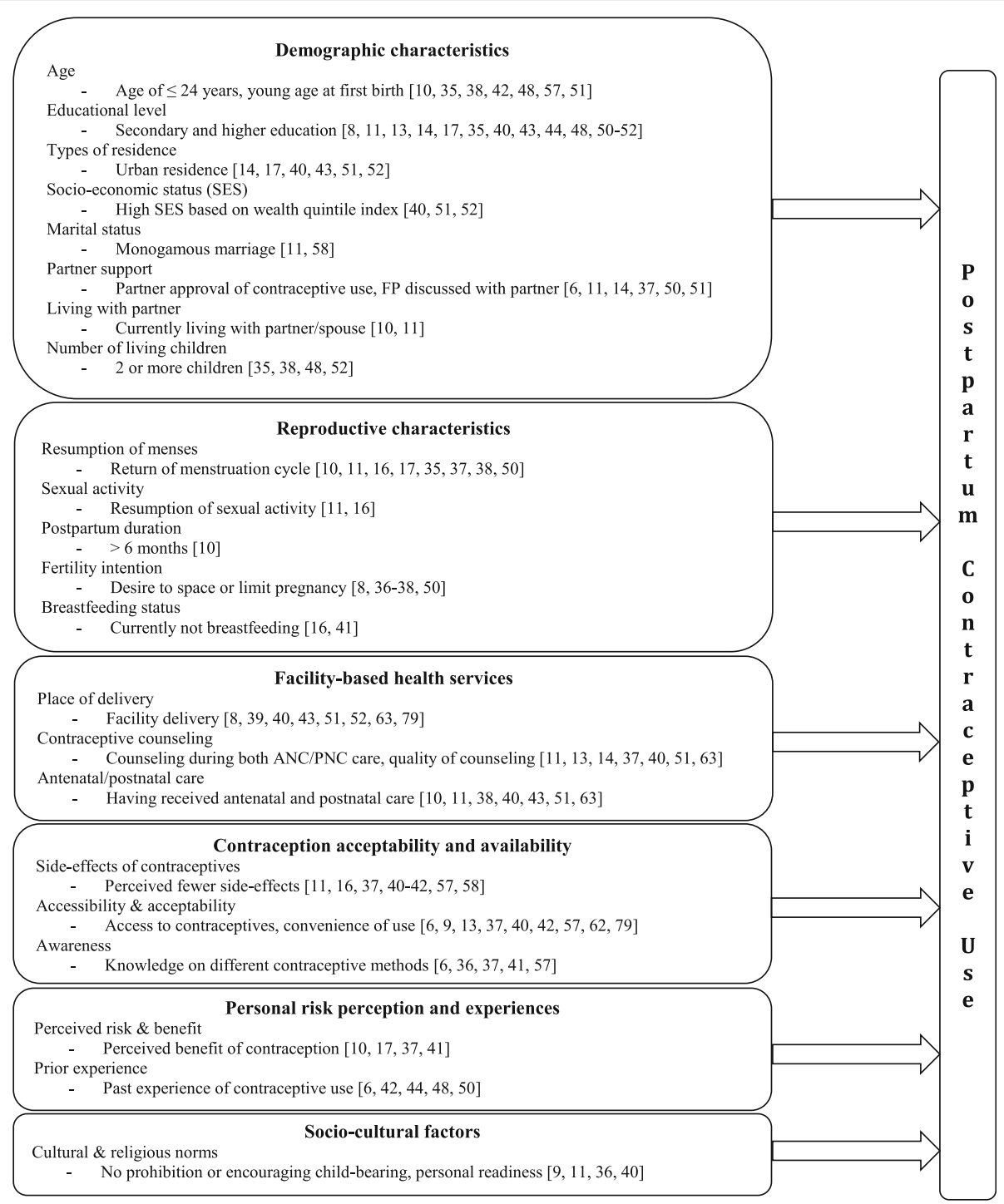

Fig. 6 Facilitators for postpartum contraceptive use, 0-12 months postpartum

on return of menses to initiate contraceptive use commonly reported in West Africa [31, 64], and fear of side-effects and concerns for partner disapproval commonly reported in East Africa [65]. However, underlying regional acceptability of contraception also contributes to these regional differences. In areas where $\mathrm{mCPR}$ is low for all women, $\mathrm{mCPR}$ among postpartum women is likely to also be low. Increasing $\mathrm{mCPR}$ in those areas will require changing social norms, demand generation for $\mathrm{FP}$, and infrastructure investments [66]. In contrast, countries with higher mCPR (South Asia/ South East Asia, East Africa) are better poised to increase contraceptive use for postpartum women specifically, through strategies such as immediate PPFP (following delivery) and expanding the range of methods available [66].

While injectables were universally the most common contraceptive method initiated during the postpartum period (followed by OCPs and condoms) in our review, the contraceptive method mix is changing in some areas. We found LARC use has dramatically increased over time in East Africa (Kenya and Ethiopia), predominately due to higher implant use, leading to lower proportions of contraceptive users selecting injectables $(68.5 \%$ in 2013 versus $34.2 \%$ in 2015 in Ethiopia). Regional changes in postpartum LARC use echo recent trends observed among nonpostpartum woman [67], which have shown increases in implant use among FP users from $\leq 2 \%$ to $\geq 10 \%$ in as few as 6 years [67]. IUD use has also risen slightly, but absolute numbers remain small. In addition, recent data suggest implant use is higher among parous women, which may begin with initiation of implants in the postpartum period. Increases in implant use may be attributed to appealing method characteristics (i.e., rapid return to fertility, ease of 
insertion, user independence, and concealability), widened eligibility for implant users - including immediately postpartum [68], and reductions in cost and increased availability due to international donor and FP2020 commitments $[67,69,70]$. Several FP2020 countries with higher mCPR have specifically identified improving PPFP as one of their primary areas of focus, including offering implants immediately postpartum. These efforts will likely have a significant impact on improving PPFP and may have significant impacts on improving birth spacing and limiting due to the long-acting, user independent properties of implants.

Strategies that have previously been successful on improving PPFP include providing multiple antenatal counseling sessions on FP at health facilities in Nigeria [63] and educational campaigns in India [71]. The educational campaign in India was unique in that it used a socioecological approach to provide education to pregnant women, mother-in-law's (or oldest female members of the family), and male partners using community health workers. By integrating culturally appropriate, educational material within the existing government program in India, women not only were more likely to understand healthy birth spacing, but were also nearly twice as likely to use a contraceptive method by 9 months postpartum [71]. It is essential for healthcare providers to support postpartum women who want to prevent or delay future pregnancies, ensuring they receive enough information on postpartum contraceptive methods and addressing individual beliefs and values about FP use in the postpartum period. Hence, adopting tailored counseling interventions using community health workers could be an effective strategy for enhancing PPFP use.

While postpartum women have unique barriers to using contraception, they also share many similarities with nonpostpartum women. Fear of side effects was reported in several studies $[11,16,37,40-42,57,58]$ in our review, and was consistently cited as a formidable barrier that led to low uptake and high discontinuation rates among postpartum women. These findings are similar to several other studies among non-postpartum women that have also shown side-effects are a barrier to contraceptive use [72, 73]. In West Africa where mCPR is low among all women, fear of side effects was the leading reason for non-use of modern contraception $[74,75]$. Since many women are engaged in the healthcare system during pregnancy and the postpartum period (often during several visits), there are opportune times to integrate contraceptive counseling into $\mathrm{MCH}$ care visits. Antenatal and postnatal contraceptive counseling should both address concerns about side effects and identify methods that align with individual values and preferences to help women who desire birth spacing and limiting achieve their desired family size.

The regional differences in the use of PPFP we found in this review suggest context specific approaches to meeting contraceptive needs during the postpartum period should be considered when developing or implementing interventions for programs. In countries where a greater proportion of postpartum women deliver at home and $\mathrm{mCPR}$ is low in the postpartum period, community-based interventions may need to be prioritized as these women might not be reached through facility-based interventions [76]. In contrast, integrating $\mathrm{FP}$ services into the $\mathrm{MCH}$ continuum of care may be a successful approach in countries where facility delivery rates are higher. This approach would offer multiple opportunities to reach women with FP information and services [77].

Our systematic review and meta-analysis had several strengths. The meta-analysis included multiple indicators on contraception, including $\mathrm{mCPR}$, unmet need, and fertility intentions, and results were disaggregated by region. The focus of our review and meta-analysis was the first year postpartum, during which most PPFP interventions are targeted [78]. Our analysis was also subject to some limitations. Publications that were not in English or did not have available full-text versions were excluded and could bias findings. The number of studies with complete data was small and may limit our ability to detect differences. Furthermore, studies included in the review and meta-analysis included heterogeneous research methodologies, with different study designs, follow-up periods (for longitudinal studies), and time-points of outcome ascertainment. Since contraceptive needs and use can change over the course of the postpartum period, our analyses may not reflect these changes over time. However, we did not detect significant differences based on timing of postpartum initiation reported. We also did not detect temporal changes in contraceptive use or unmet need for contraception, but may lack power to detect these heterogeneity.

\section{Conclusions}

PPFP use among the women during the first year after delivery was low and desire for birth spacing and birth limiting was high in LMICs. A global increase in uptake of PPFP can help women establish healthy birth spacing and limiting and reduce adverse $\mathrm{MCH}$ outcomes. Potential strategies to increase $\mathrm{mCPR}$ among postpartum women who want to delay or prevent future pregnancies include adopting tailored counseling approaches and providing accurate information on the range of FP methods, through community-based intervention programs. Developing new counseling strategies and policies to support counseling at multiple points along the pregnancy-postpartum continuum may help improve PPFP. Finally, segmented approaches to supporting PPFP may be effective in reducing unmet need in the early postpartum periods, improving method satisfaction, and reducing discontinuation rates among women who intend to space or limit future pregnancies. 


\section{Appendix}

\section{a}

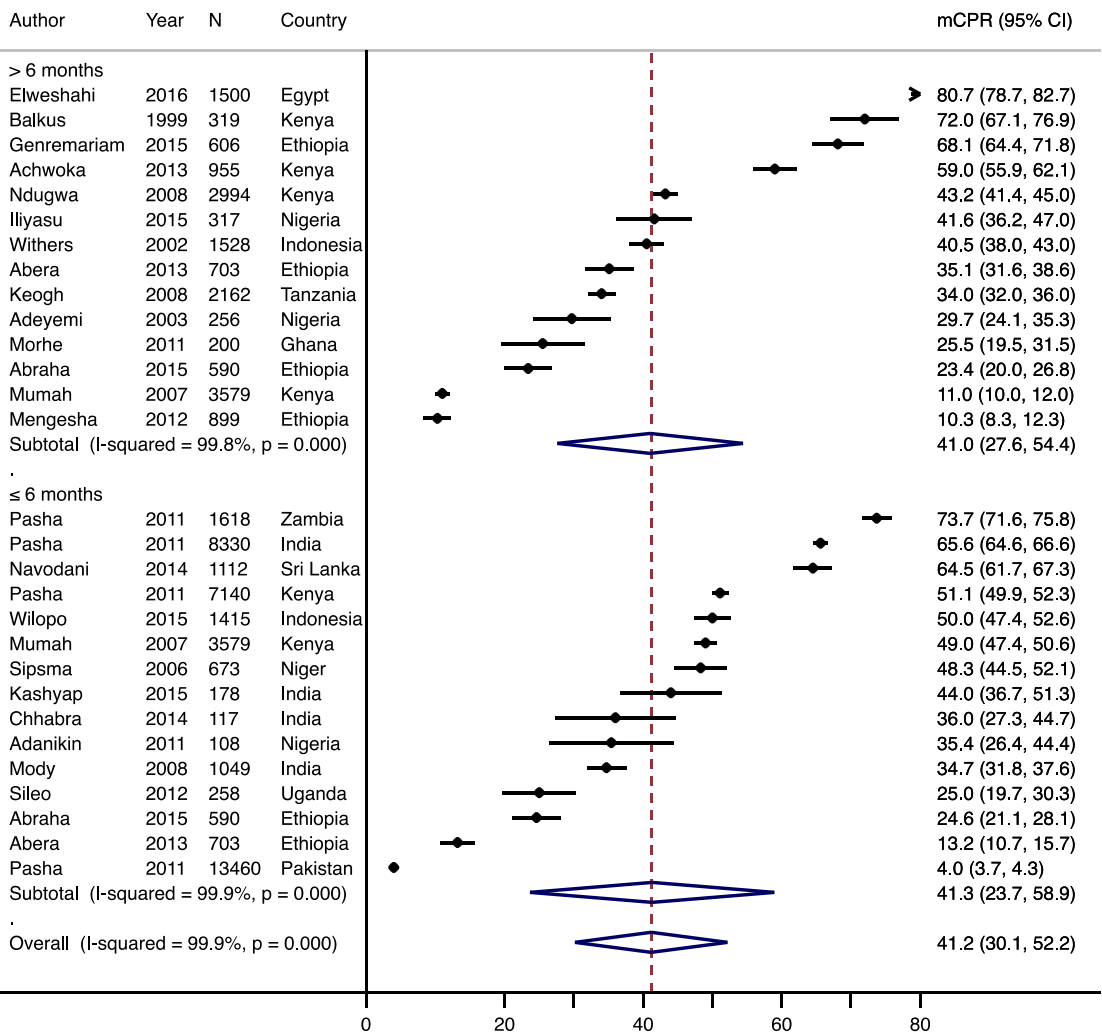

b
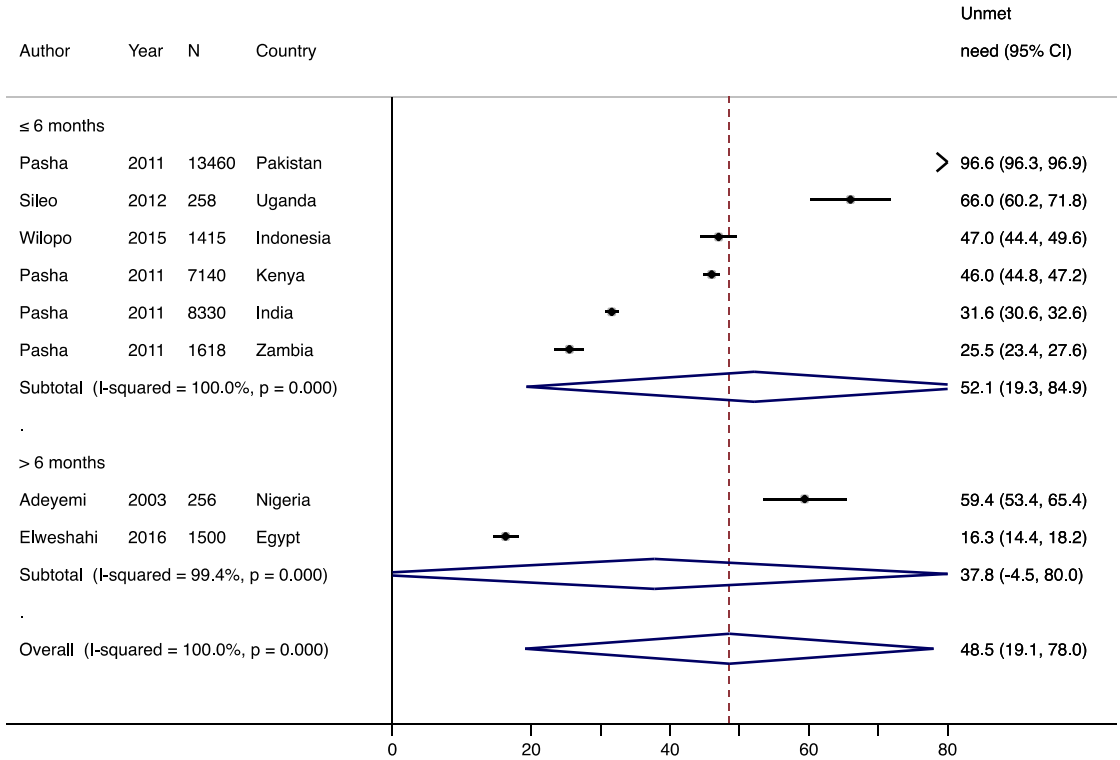

Fig. 7 a. Forest plot of modern contraceptive prevalence rate (mCPR), by postpartum follow-up period. Year is start of study and $\mathrm{N}$ is sample size. mCPR; modern contraceptive prevalence rate, Cl; confidence interval. $\mathbf{b}$. Forest plot for unmet need for contraception, by postpartum follow-up period. Year is start of study and $\mathrm{N}$ is sample size. Cl; confidence interval 


\section{Abbreviations}

ANC: Antenatal care; Cl: Confidence interval; DHS: Demographic and Health Survey; ECP: Emergency contraceptive pill; FP: Family planning; HIV: Human Immunodeficiency Virus; IUD: Intrauterine device; LAM: Lactational amenorrhea method; LARC: Long-acting reversible contraceptive; MCH: Maternal and child health; mCPR: Modern contraceptive prevalence rate; OCP: Oral contraceptive pill; OR: Odds ratio; PPFP: Postpartum family planning; PRISMA: Preferred reporting items for systematic reviews and meta-analyses; PNC: Postnatal care; SSA: sub-Saharan Africa

\section{Acknowledgements}

The authors would like to thank Joanne Rich and Sarah Safranek, librarians at the University of Washington Health Sciences Library for their help in developing the search strategy used in the review.

\section{Author's contributions}

$\mathrm{RD}$ and $\mathrm{AD}$ developed the concept for the manuscript. RD drafted the manuscript and RD and MF conducted the review and RD conducted all analyses. AD, PK, JU, and NFW provided detailed comments on the draft that had been submitted. All authors contributed to reviewing and revising the manuscript and approved the final version.

\section{Funding}

The University of Washington's Global Center for Integrated Health of Women, Adolescents, and Children (Global WACh) and NIH/NIAID K01 Al116298 to ALD supported work on this project.

\section{Availability of data and materials}

All relevant data are within the paper. Additional data are available from the corresponding author on reasonable request.

\section{Ethics approval and consent to participate}

Not applicable.

\section{Consent for publication}

Not applicable.

\section{Competing interests}

The authors declare that they have no competing interests.

\section{Author details \\ 'Sun Yat-sen Global Health Institute, Sun Yat-sen University, Guangzhou, Guangdong, China. ${ }^{2}$ Department of Psychosocial and Community Health \& Department of Global Health, University of Washington, Seattle, WA, USA. ${ }^{3}$ Cardea Services, Seattle, WA, USA. ${ }^{4}$ Department of Obstetrics and Gynecology, University of Washington, Seattle, WA, USA. ${ }^{5}$ Department of Biobehavioral Nursing and Health Informatics, University of Washington, Seattle, WA, USA. 'Department of Global Health, University of Washington, Seattle, WA, USA.}

Received: 8 January 2019 Accepted: 9 October 2019

Published online: 29 October 2019

\section{References}

1. Appareddy S, Pryor J, Bailey B. Inter-pregnancy interval and adverse outcomes: evidence for an additional risk in health disparate populations. J Matern Fetal Neonatal Med. 2017;30:2640-4.

2. Marston C. Report of a WHO technical consultation on birth spacing Geneva Switzerland 13-15 June 2005; 2006.

3. Rutstein SO. Effects of preceding birth intervals on neonatal, infant and under-five years mortality and nutritional status in developing countries: evidence from the demographic and health surveys. Int J Gynecol Obstet. 2005;89:S7-S24.

4. WHO: Postpartum family planning: essential for ensuring health of women and their babies. World Contraception Day 2018. Accessed on 13 Sept 2019 at https://www.who.int/reproductivehealth/topics/family_planning/worldcontraception-day-2018/en/.

5. WHO: Programming strategies for postpartum family planning. ISBN 97892 41506496 (NLM classification: WA 550). Geneva: World Health Organization. 2013.
6. Eliason S, Baiden F, Quansah-Asare G, Graham-Hayfron Y, Bonsu D, Phillips J, Awusabo-Asare K. Factors influencing the intention of women in rural Ghana to adopt postpartum family planning. Reprod Health. 2013;10:34.

7. Prata N, Passano P, Rowen T, Bell S, Walsh J, Potts M. Where there are (few) skilled birth attendants. J Health Population Nutr. 2011;29:81.

8. Pasha O, Goudar SS, Patel A, Garces A, Esamai F, Chomba E, Moore JL, Kodkany BS, Saleem S, Derman RJ. Postpartum contraceptive use and unmet need for family planning in five low-income countries. Reprod Health. 2015;12:1.

9. Ross JA, Winfrey WL. Contraceptive use, intention to use and unmet need during the extended postpartum period. Int Fam Plan Perspect. 2001:20-7.

10. Abera Y, Mengesha ZB, Tessema GA. Postpartum contraceptive use in Gondar town, Northwest Ethiopia: a community based cross-sectional study. BMC Womens Health. 2015:15:19.

11. Abraha TH, Teferra AS, Gelagay AA. Postpartum modern contraceptive use in northern Ethiopia: prevalence and associated factors. Epidemiol Health. 2017;39

12. Amelya $\mathrm{M}$, Andriyana $\mathrm{H}$, Nababan $\mathrm{B}$, Rusdianto E. Postpartum contraception in Indonesian teenager. KnE Medicine. 2016;1:81-8.

13. Chhabra HK, Mohanty IR, Mohanty NC, Thamke P, Deshmukh YA. Impact of structured counseling on choice of contraceptive method among postpartum women. J Obstetrics Gynecol India. 2016;66:471-9.

14. Kashyap C, Mohanty IR, Thamke P, Deshmukh YA. Acceptance of contraceptive methods among postpartum women in a tertiary care center. J Obstetr Gynecol India. 2017;67:91-7.

15. Harrison MS, Goldenberg RL. Immediate postpartum use of long-acting reversible contraceptives in low-and middle-income countries. Matern Health Neonatol Perinatol. 2017;3:24.

16. Moore Z, Pfitzer A, Gubin R, Charurat E, Elliott L, Croft T. Missed opportunities for family planning: an analysis of pregnancy risk and contraceptive method use among postpartum women in 21 low-and middle-income countries. Contraception. 2015;92:31-9.

17. Ndugwa RP, Cleland J, Madise NJ, Fotso J-C, Zulu EM. Menstrual pattern, sexual behaviors, and contraceptive use among postpartum women in Nairobi urban slums. J Urban Health. 2011:88:341-55.

18. The World Bank. World Bank Countries and Lending Groups. URL: https:// datahelpdesk.worldbank.org/knowledgebase/articles/906519-world-bankcountry-and-lending-groups. Accessed 21 October 2018.

19. Stang A. Critical evaluation of the Newcastle-Ottawa scale for the assessment of the quality of nonrandomized studies in meta-analyses. Eur 」 Epidemiol. 2010;25:603-5.

20. Festin MPR, Kiarie J, Solo J, Spieler J, Malarcher S, Van Look PFA Temmerman M. Moving towards the goals of FP2020_classifying contraceptives. Contraception. 2016:94:289-94.

21. Hubacher D, Trussell J. A definition of modern contraceptive methods Contraception. 2015;92:420-1.

22. Hindin MJ, Kalamar AM. Country-specific data on the contraceptive needs of adolescents. Bull World Health Organ. 2017;95:166.

23. Scoggins S, Bremner J. FP2020 momentum at the midpoint 2015-2016; 2016.

24. Track20: The S-Curve: Putting mCPR Growth in Context. Available at http:// www.track20.org/download/pdf/S_Curve_One_Pager.pdf. 2017. Accessed 21 October 2018.

25. Van Lith LM, Yahner M, Bakamjian L. Women's growing desire to limit births in sub-Saharan Africa: meeting the challenge. Global Health. 2013;1:97-107.

26. Cleland J, Shah $H_{H}$, Benova L. A fresh look at the level of unmet need for family planning in the postpartum period, its causes and program implications. Int Perspect Sex Reprod Health. 2015;41:155-62.

27. Rossier C, Bradley SEK, Ross J, Winfrey W. Reassessing unmet need for family planning in the postpartum period. Stud Fam Plan. 2015;46:355-67.

28. Higgins JPT, Thompson SG, Deeks JJ, Altman DG. Measuring inconsistency in meta-analyses. Bmj. 2003;327:557-60.

29. Borenstein $M$, Hedges LV, Higgins J, Rothstein HR. A basic introduction to fixed-effect and random-effects models for meta-analysis. Res Synth Methods. 2010;1:97-111.

30. Berkey CS, Hoaglin DC, Mosteller F, Colditz GA. A random-effects regression model for meta-analysis. Stat Med. 1995;14:395-411.

31. Tsui AO, Brown W, Li Q. Contraceptive practice in sub-Saharan Africa. Popul Dev Rev. 2017:43:166-91.

32. Van der Wijden C, Manion C. Lactational amenorrhoea method for family planning. Cochrane Database Syst Rev. 2015. 
33. World Health O: Exclusive breastfeeding for optimal growth, development and health of infants. World Health Organization 2017. Accessed 26 Oct 2019.

34. Dallal GE: The Standard Error of a Proportion. Available at http://www. jerrydallal.com/lhsp/psd.htm. 1998.

35. Mumah JN, Machiyama K, Mutua M, Kabiru CW, Cleland J. Contraceptive adoption, discontinuation, and switching among postpartum women in Nairobi's urban slums. Stud Fam Plan. 2015;46:369-86.

36. O'Shea MS, Rosenberg NE, Hosseinipour MC, Stuart GS, Miller WC, Kaliti SM, Mwale M, Bonongwe PP, Tang JH. Effect of HIV status on fertility desire and knowledge of long-acting reversible contraception of postpartum Malawian women. AIDS Care. 2015;27:489-98.

37. Robinson N, Moshabela M, Owusu-Ansah L, Kapungu C, Geller S. Barriers to intrauterine device uptake in a rural setting in Ghana. Health Care Women Int. 2016:37:197-215

38. Withers M, Kano M, Pinatih GNI. Desire for more children, contraceptive use and unmet need for family planning in a remote area of Bali, Indonesia. J Biosoc Sci. 2010;42:549-62.

39. MCHIP: Maternal and Child Health Integrated Program. Kenya 2008-2009 DHS Reanalysis for PPFP. Family Planning Needs during the First Two Years Postpartum in Kenya.; 2012.

40. International Seminar Report. Promoting Postpartum and Post-Abortion Family Planning: Challenges and Opportunities. Cochin, India, 11-13. 2014

41. Adeyemi AB, ljadunola KT, Orji EO, Kuti O, Alabi MM. The unmet need for contraception among Nigerian women in the first year post-partum. Eur J Contracept Reprod Health Care. 2005;10:229-34.

42. Hubacher D, Masaba R, Manduku CK, Veena V. Uptake of the levonorgestrel intrauterine system among recent postpartum women in Kenya: factors associated with decision-making. Contraception. 2013;88:97-102.

43. Mengesha ZB, Worku AG, Feleke SA. Contraceptive adoption in the extended postpartum period is low in Northwest Ethiopia. BMC pregnancy and childbirth. 2015;15:160

44. Sileo KM, Wanyenze RK, Lule H, Kiene SM. Determinants of family planning service uptake and use of contraceptives among postpartum women in rural Uganda. Int j Public Health. 2015;60:987-97.

45. Elweshahi HMT, Gewaifel Gl, Sadek SSELD, El-Sharkawy OG: Unmet need for postpartum family planning in Alexandria, Egypt. Alexandria Journal of Medicine 2017.

46. Wulifan JK, Brenner S, Jahn A, De Allegri M. A scoping review on determinants of unmet need for family planning among women of reproductive age in low and middle income countries. BMC Womens Health. 2015;16:2.

47. Sedgh $G$, Hussain R. Reasons for contraceptive nonuse among women having unmet need for contraception in developing countries. Stud Fam Plan. 2014:45:151-69.

48. Mody SK, Nair S, Dasgupta A, Raj A, Donta B, Saggurti N, Naik DD, Silverman JG. Postpartum contraception utilization among low-income women seeking immunization for infants in Mumbai, India. Contraception. 2014;89:516-20.

49. Wilopo SA, Setyawan A, Pinandari AW, Prihyugiarto T, Juliaan F, Magnani RJ. Levels, trends and correlates of unmet need for family planning among postpartum women in Indonesia: 2007-2015. BMC Womens Health. 2017;17:120.

50. Keogh SC, Urassa M, Kumogola Y, Kalongoji S, Kimaro D, Zaba B. Postpartum contraception in northern Tanzania: patterns of use, relationship to antenatal intentions, and impact of antenatal counseling. Stud Fam Plan. 2015;46:405-22

51. Sipsma HL, Bradley EH, Chen PG. Lactational amenorrhea method as a contraceptive strategy in Niger. Matern Child Health J. 2013;17:654-60.

52. Hounton S, Winfrey W, Barros AJD, Askew I. Patterns and trends of postpartum family planning in Ethiopia, Malawi, and Nigeria: evidence of missed opportunities for integration. Glob Health Action. 2015;8: 29738.

53. Iliyasu Z, Galadanci HS, Danlami KM, Salihu HM, Aliyu MH. Correlates of postpartum sexual activity and contraceptive use in Kano, northern Nigeria. Afr J Reprod Health. 2018;22:103-12.

54. Morhe ESK, Ankobea F, Asubonteng GO, Opoku B, Turpin CA, Dalton VK: Postpartum contraceptive choices among women attending a well-baby clinic in Ghana. International Journal of Gynecology \& Obstetrics. 2017;138:219-224.
55. Achwoka D, Pintye J, McGrath CJ, Kinuthia J, Unger JA, Obudho N, Langat A, John-Stewart G, Drake AL. Uptake and correlates of contraception among postpartum women in Kenya: results from a national cross-sectional survey. Contraception. 2017:97:227-35.

56. Navodani KT, Fonseka P, Goonewardena CS. Postpartum family planning: missed opportunities across the continuum of care. Ceylon Med J. 2017;62:87-91

57. Shabiby MM, Karanja JG, Odawa F, Kosgei R, Kibore MW, Kiarie JN, Kinuthia J. Factors influencing uptake of contraceptive implants in the immediate postpartum period among HIV infected and uninfected women at two Kenyan District hospitals. BMC Womens Health. 2015;15:62.

58. Balkus J, Bosire R, John-Stewart G, Mbori-Ngacha D, Schiff MA, Wamalwa D, Gichuhi C, Obimbo E, Wariua G, Farquhar C. High uptake of postpartum hormonal contraception among HIV-1-seropositive women in Kenya. Sex Transm Dis. 2007:34:25.

59. Gebremariam A, Gebremariam H. Contraceptive use among lactating women in Ganta-Afeshum District, eastern Tigray, northern Ethiopia, 2015: a cross sectional study. BMC Pregnancy Childbirth. 2017;17:421.

60. Gebremedhin AY, Kebede Y, Gelagay AA, Habitu YA. Family planning use and its associated factors among women in the extended postpartum period in Addis Ababa, Ethiopia. Contracept Reprod Med. 2018;3:1.

61. Truitt ST, Fraser AB, Grimes DA, Gallo MF, Schulz KF. Hormonal contraception during lactation: systematic review of randomized controlled trials. Contraception. 2003;68:233-8.

62. Mohammed-Durosinlorun A, Abubakar A, Adze J, Bature S, Mohammed C, Taingson M, Ojabo A. Comparison of contraceptive methods chosen by breastfeeding, and non-breastfeeding, women at a family planning clinic in northern Nigeria. Health. 2016;8:191.

63. Adanikin Al, Onwudiegwu U, Loto OM. Influence of multiple antenatal counselling sessions on modern contraceptive uptake in Nigeria. Eur J Contracept Reprod Health Care. 2013;18:381-7.

64. Iliyasu Z, Kabir M, Galadanci HS, Abubakar IS, Salihu HM, Aliyu MH. Postpartum beliefs and practices in Danbare village, northern Nigeria. J Obstet Gynaecol. 2006;26:211-5.

65. Keesara S, Juma PA, Harper CC, Newmann SJ. Barriers to postpartum contraception: differences among women based on parity and future fertility desires. Culture Health Sexuality. 2018;20:247-61.

66. Track20: The S-Curve: Putting mCPR Growth into Context. Accessed on June 4, 2018 at http://www.track20.org/pages/data_analysis/in_depth/mCPR_ growth/s_curve.php. 2017.

67. Jacobstein R: Liftoff: The Blossoming of Contraceptive Implant Use in Africa. Global Health: Science and Practice 2018:GHSP-D.

68. World Health O: Medical eligibility criteria for contraceptive use. Geneva: WHO; 2015. World Health Organization 2017.

69. FP2020: Bayer halves the price of its contraceptive implant Jadelle for women in developing countries. Accessed on Sept 30, 2018 at http://www. familyplanning2020.org/articles/12388. 2016. Accessed 30 Sept 2018.

70. (FP2020) FP: FP2020 Annual commitment update questionnaire response. MERCK (MSD). http://ec2-54-210-230-186.compute-1.amazonaws.com/wpcontent/uploads/2016/09/FP2020_2016_Annual_Commitment_Update_ Questionnaire-Merck_DLC.pdf. 2016. Accessed 30 Sept 2018.

71. Sebastian MP, Khan ME, Kumari K, Idnani R. Increasing postpartum contraception in rural India: evaluation of a community-based behavior change communication intervention. Int Perspect Sex Reprod Health. 2012:68-77.

72. Sedgh G, Ashoford LS, Hussain R: Unmet need for contraception in developing countries: examine women's reasons for not using a method. New York: The Guttmacher Institute; 2016.

73. Aviisah MA, Norman ID, Enuameh Y. Facilitators and barriers to modern contraception use among reproductive-aged women living in sub-Saharan Africa: a qualitative systematic review protocol. JBI Database System Rev Implement Rep. 2017;15:2229-33.

74. Hindin MJ, McGough LJ, Adanu RM: Misperceptions, misinformation and myths about modern contraceptive use in Ghana. J Fam Plann Reprod Health Care 2013:ffprhc-2012.

75. Asekun-Olarinmoye EO, Adebimpe WO, Bamidele JO, Odu OO, AsekunOlarinmoye 10, Ojofeitimi EO. Barriers to use of modern contraceptives among women in an inner city area of Osogbo metropolis, Osun state, Nigeria. Int J Womens Health. 2013;5:647.

76. Track20. Access: PPFP. Published on July 2017. Accessed at http://www. track20.org/pages/data_analysis/in_depth/opportunities/PPFP.php. Accessed 30 Sept 2018 
77. Gatto AB: Family Planning in the Continuum of Care: The Postpartum and Post-abortion Connection. Accessed at https://medium.com/@FP202

OGlobal_20685/family-planning-in-the-continuum-of-care-the-postpartumand-post-abortion-connection-7d81a590fec2 on September 2018.

78. Blazer C, Prata N. Postpartum family planning: current evidence on successful interventions. Open Access J Contracept. 2016;7:53-67.

\section{Publisher's Note}

Springer Nature remains neutral with regard to jurisdictional claims in published maps and institutional affiliations.

Ready to submit your research? Choose BMC and benefit from:

- fast, convenient online submission

- thorough peer review by experienced researchers in your field

- rapid publication on acceptance

- support for research data, including large and complex data types

- gold Open Access which fosters wider collaboration and increased citations

- maximum visibility for your research: over $100 \mathrm{M}$ website views per year

At $\mathrm{BMC}$, research is always in progress.

Learn more biomedcentral.com/submissions 